\title{
New Methods of Analysis and Investigation of Terpenoid Indole Alkaloids
}

\section{Guitele Dalia Goldhaber-Pasillas, Young Hae Choi ${ }^{1}$, Robert Verpoorte} Natural Products Laboratory, Institute of Biology Leiden, Leiden University, Leiden, The Netherlands ${ }^{1}$ Corresponding author: e-mail address: y.choi@chem.leidenuniv.nl

\section{Contents}

1. Introduction 235

2. Extraction and Purification Methods 236

$\begin{array}{ll}2.1 & \text { Solvent extraction } \\ 2.2 & 237\end{array}$

2.2 lonic liquids 239

$\begin{array}{ll}2.3 \text { Molecular imprinted polymers } & 239\end{array}$

$\begin{array}{ll}2.4 \text { Supercritical } \mathrm{CO}_{2} & 240\end{array}$

3. Analytical Methods 242

3.1 HPLC and hyphenated techniques 242

$\begin{array}{ll}\text { 3.2 Ultra high-pressure LC-MS } & 243\end{array}$

3.3 Gas chromatography coupled to mass spectroscopy 248

3.4 High-speed counter-current chromatography 251

$\begin{array}{ll}3.5 & \text { Capillary electrophoresis } 253\end{array}$

3.6 Quantitative nuclear magnetic resonance 259

4. Applications in Fingerprint Analysis 260

4.1 NMR and LC-MS-based metabolic fingerprinting on TIAs 260

5. Conclusions 262

References 263

\begin{abstract}
Terpenoid indole alkaloids are biologically active compounds that have been used as pharmaceuticals among others as anticancer, antimalarial, antihypertensive and hypoglycemic agents for more than 40 years. Many efforts have been focused on their extraction, isolation, separation and structural elucidation by means of different approaches based on methodologies already established since the 1960s. New methodologies in extraction and sample preparation from different matrices include environmentally
\end{abstract}


friendly techniques such as ionic liquids or supercritical fluid extraction as well as the synthesis of molecularly imprinted polymers. Chromatography combined with spectroscopy is still the preferred analytical tool for alkaloid analysis, but recent improvements in mass spectroscopy and nuclear magnetic resonance-based technologies have also been applied to areas of research such as toxicology, quality control, metabolic fingerprinting and metabolic profiling. This review is intended to provide a more general rather than an exhaustive overview on the new methods for terpenoid indole alkaloid analysis focusing on hyphenated high-performance liquid chromatography and mass spectrometry approaches. We will also discuss extraction methods and the strength and weaknesses of the different analytical tools for their application in targeted or non targeted approaches.

\section{ABBREVIATIONS}

APCI atmospheric pressure chemical ionization

CCC counter-current chromatography

CE capillary electrophoresis

CID collision-induced dissociation

CPC centrifugal partition chromatography

CZE capillary zone electrophoresis

DAD photodiode array detection

ESI electrospray ionization

FID flame ionization detection

GC-MS gas chromatography coupled to mass spectroscopy

HPCCC high-performance counter-current chromatography

HPLC high-performance liquid chromatography

HSCCC high-speed counter-current chromatography

IT ion trap

LC-MS liquid chromatography coupled to mass spectroscopy

LOD limit of detection

MEKC micellar electrokinetic chromatography

MIP molecularly imprinted polymer

MS mass spectrometry

$\mathbf{M S}^{\mathbf{n}}$ multistage mass (tandem mass) spectroscopy

NACE non-aqueous capillary electrophoresis

NMR nuclear magnetic resonance

qNMR quantitative nuclear magnetic resonance

SFE supercritical fluid extraction

SIM single ion monitoring

TIAs terpenoid indole alkaloids

TOF time of flight

UHPLC ultra high-pressure liquid chromatography

UV ultraviolet 


\section{INTRODUCTION}

Terpenoid indole alkaloids (TIAs) are a group of ca. 3000 natural products among which a large number of compounds are being applied as medicine in pharmacology such as vinblastine, vincristine, strychnine and reserpine. Consequently, over the years, a lot of work has been done in bioprospecting for novel drugs from this group, and to improve the production of these compounds in plants or genetically modified organisms. All these studies require analytical tools to identify and quantify these compounds in various matrices such as plants and biological fluids. These tools are mainly based on chromatography and spectroscopy and different combinations of these. Thin-layer chromatography (TLC), gas chromatography (GC) (both since the 1960s) and high-performance liquid chromatography (HPLC; since the 1970s) offer efficient methods for the separation and identification of alkaloids based on retention behaviour that is further supported by more or less specific detection methods. Specificity in detection for TLC is obtained by colour reactions and ultraviolet (UV) spectroscopy, for GC by the combination with mass spectrometry (MS) and for HPLC by UV, MS and nuclear magnetic resonance (NMR) spectroscopy. Ever since the introduction of these methods, they have been extensively applied for alkaloids; however, in terms of separation principle, not much has changed since the first decade of use of these chromatographic tools (Baerheim Svendsen \& Verpoorte, 1983; Verpoorte \& Baerheim Svendsen, 1984). Stationary phases have improved, but the principle of retention of alkaloids has not changed, for example, in HPLC this is mainly reversed-phase chromatography with slightly acidic solvents, sometimes containing modifiers to reduce tailing due to adsorption of alkaloids on residual silanol groups in the stationary phase, or using ion-pair chromatography to change the selectivity. Though it is always claimed that the a new stationary phase has many advantages and is much better than the previous ones, the major disadvantage is that a different chromatogram will be obtained, requiring extensive validation, and direct comparison with previous results is not possible anymore.

This is where spectroscopic methods without hyphenation with chromatography have an advantage, as they do not involve a separation step. In the case of UV, infrared and NMR, the detection mechanism is based on pure physical properties, in case of a pure compound showing spectra specific for 
the chemical structure, in case of mixtures the sum of the spectra of the compounds present. In case of NMR spectroscopy, two-dimensional (2D) NMR can be applied to deconvolute the spectra of the compounds present. NMR has one major advantage above all methods and that is in ${ }^{1} \mathrm{H}$ NMR, all signals can be directly compared. The total signal intensity of each proton is only dependent on the molar concentration of the compound it is part of, which means that one can, without the need for individual calibration curves, quantify the compounds present in a mixture. In the case of mass spectroscopy, a manipulation is required to ionize the compound(s) present, which is a weakness as the spectra obtained are dependent on the instrumental conditions, as well as on the matrix. The advantage is that the resolution, is on the level of the molecular weight and in high-resolution mode, even the molecular formula can be obtained. The possibility of measuring fragmentation of the compounds adds further information for the identity of the compound.

In recent years combining the spectroscopy with chromatography has become common practice; thus, combining the advantages of both is particularly of great value in the identification of unknown, possibly novel, compounds. However, for the quantitative analysis of known compounds, liquid chromatography coupled to UV (LC-UV) and gas chromatography coupled to flame ionization detector (GC-FID) in many cases are fitting the needs of a quantitative analysis, as they are robust and reproducible methods.

\section{EXTRACTION AND PURIFICATION METHODS}

An elemental aspect in any analytical methodology is the compatibility of the extraction method with the analytical system, which will be ultimately reflected in the quality and usefulness of the chromatograms or spectra. Conventionally, extraction procedures for alkaloids have been done by different mechanical, physical and chemical processes such as Soxhlet extraction, maceration or percolation using organic solvents and liquid-liquid partitioning including aqueous solutions, although nowadays non-conventional methods which are more friendly to the environment have been successfully applied to alkaloid extraction such as ultrasound, microwave, supercritical fluids and ionic liquids (ILs).

The extraction has several aspects that need to be considered: the stability of compounds, the solubility and dissolution rate and the suitability of the extract for the analytical method. The stability is of concern from the very first moment of sampling, for example, when a plant is harvested, immediately the stress metabolism will start which may affect the alkaloids levels, for 
example, catabolism, de novo biosynthesis and (enzymatic) hydrolysis. Quenching of all metabolic activities is thus required, which can be achieved by heating or even treating with microwaves or freezing followed by freezedrying. Moreover, the extraction procedure itself can cause the formation of artefacts (Maltese, van der Kooy, \& Verpoorte, 2009; Verpoorte, Choi, Mustafa, \& Kim, 2008) through reactivity of solvents or contaminations in solvents or by decomposition by heat (e.g. Soxhlet). Solubility can be good, but the rate of solubilization can be slow. Thus, extraction time is an important factor, as is the temperature; a balance must be found between these two in connection with the stability of the compounds to be extracted. In HPLC, the sample must be injected into a solvent that is similar to the mobile phase, for example, a chloroform extract cannot be directly injected on a reversed-phase column. In GC, the solvent must be volatile; in TLC, dirty extracts are not a problem as long as they do not interfere with the separation as a plate is only used once. Therefore, in every extraction procedure, such considerations must be made and it is important to properly validate the extraction method applied in any analytical protocol.

\subsection{Solvent extraction}

Extraction and sample preparation are the first key steps in plant analysis. Any analysis requires the extraction of the desired product from a complex matrix, dissolve it into the appropriate solvent and remove all the undesired compounds that might interfere with the analysis. TIAs have a lipophilic character as free bases at high $\mathrm{pH}$ and can be extracted with organic solvents such as alcohols, chloroform or ethyl acetate after plant material is basified with ammonia or sodium carbonate. A too high $\mathrm{pH}$ should be avoided as in that case phenolic alkaloids might not be fully extracted. Alternatively, alkaloids can be extracted with polar solvents at low $\mathrm{pH}$, for example, water acidified with phosphoric acid (Moreno, van der Heijden, \& Verpoorte, 1993), acetic acid (de Castro et al., 2012; Girardot et al., 2012; Jenks, 2002; Tanaka et al., 2007), tartaric acid (Jenett-Siems, Weigl, Kaloga, Schulz, \& Eich, 2003), trifluoroacetic acid (Silvestrini et al., 2002) or hydrochloric acid (Andrade et al., 2005; Verma, Laakso, Seppanen-Lakso, Huhtikangas, \& Riekkola, 2007). TIAs have also been extracted without adding acid or base with methanol (Paranhos, Fragoso, da Silveira, Henriques, \& Fett-Neto, 2009; Sun \& Liu, 2008), dichloromethane (Kumar, Bulumulla, Wimalasiri, \& Reisch, 1994), acetone (Zhang, Yu, Liu, \& Liu, 2007), ethanol (Wang et al., 2005), methanol-water 
(Wang et al., 2012), chloroform or petrol (Etse, Gray, Thomas, \& Waterman, 1989).

TIAs can be further purified by liquid-liquid extraction after basification, extraction with an immiscible organic solvent, for example, 10\% of ammonia, and then extracted with ethyl acetate (Cao et al., 2012) or from an organic solvent with an aqueous acidic solution. In such liquid-liquid partitioning methods, one should keep in mind that non-polar counter ions like chloride, acetate and trifluoroacetic acids may form ion pairs with alkaloids that are well soluble in the organic solvents (Hermans-Lokkerbol \& Verpoorte, 1986). Such approaches obviously separate alkaloids from other compounds with either non-polar (e.g. lipids) or polar character (e.g. sugars) that lack this dual lipophilic/hydrophilic character. It is therefore a targeted analysis for alkaloids.

Solid-phase extraction (SPE) involves selective extraction of TIAs from liquid samples onto a solid support using adsorption or ion exchange materials. For adsorption of TIAs in their free neutral form, reversed-phase materials such as $\mathrm{C}_{8}$ and $\mathrm{C}_{18}$ on silica are widely used. Cation exchangers can be used to selectively bind alkaloids from aqueous extracts (Sheludko, Gerasimenko, Unger, Kostenyuk, \& Stoeckgit, 1999). The choice of a SPE method also depends on the type of extract required in the analysis. For GC analysis, the alkaloids should be in the basic form, and for LC, it can be in the basic form or more commonly in the protonated form as in reversed-phase chromatography usually weakly acidic systems are applied. Depending on the method of detection, chlorine- or fluorine-containing solvents should be avoided for GC and for MS, and all components need to be volatile.

Quaternary indole alkaloids can be isolated by precipitation. A crude extract is prepared with an acidic aqueous solution and then they are precipitated with Mayer's reagent at pH 5 (Penelle et al., 2001; Verpoorte \& Baerheim Svendsen, 1984) or as Reineckate salts at $\mathrm{pH} 8$ (Ghosal \& Srivastava, 1974) or $\mathrm{pH} 3$ (Hu, Zhu, Prewo, \& Hesse, 1989). After collection, the precipitate is dissolved into a mixture of organic solvents like acetone-methanol-water 6:2:1 v/v/v (Perera, Samuelson, van Beek, \& Verpoorte, 1983; Verpoorte \& Baerheim Svendsen, 1984) and exchanged to chlorides by means of an anion exchanger. Nevertheless, because these salts pose serious health risks to humans and to the environment, their use should be discouraged. Quaternary alkaloids can be adsorbed from aqueous extracts using cation exchangers. 


\subsection{Ionic liquids}

ILs have been successfully applied to the extraction of complicated samples such as plant complexes or low-accumulation constituents like TIAs. Since the rate of solubilization in the often viscous IL is a limiting step, they are frequently used in ultrasound- or microwave-assisted extraction. Further steps to make the extract suitable for the analysis include liquid-liquid extraction, liquid-phase microextraction, solid-phase microextraction and aqueous two-phase system extraction. Particularly, microwave-assisted extraction has been the preferred option as it is a rapid, effective and cheap technique for the extraction of TIAs from different matrices (Sparr \& Bjorklund, 2000).

In an IL-based ultrasound-assisted approach for the extraction of TIAs from Catharanthus roseus, Yang et al. (2011) tested the extraction efficiency of 1-allyl-3-methylimidazolium bromide at a concentration of $0.5 \mathrm{M}$ and a solid-liquid ratio of 1:10 for $2 \mathrm{~h}$ of maceration time showing higher extraction efficiency when compared to seven different conventional extraction methods. The same cation and anion combination along with microwave-assisted extraction proved to be effective for isolating alkaloids from Camptotheca acuminata (Ma et al., 2012; Wang et al., 2011).

\subsection{Molecular imprinted polymers}

Molecularly imprinted polymers (MIPs) are an emerging technique that uses polymer materials with high selectivity and affinity towards a particular molecule, the template. MIPs are prepared by crosslinking monomers in a complexation solution in the presence of the template molecules. After the polymerization reaction is finished, the template is removed from the polymer by solvent extraction, leaving behind an imprint with a cavity that sterically and chemically binds the template molecule with high affinity (Xie et al., 2001).

Highly selective MIPs were used in SPE for preparative and analytical separations, using, for example, HPLC to separate vinblastine (Zhu, Huang, Li, \& Yin, 2010), vindoline and catharanthine (Lopez et al., 2011) from a commercial extract of C. roseus. Methacrylic acid was used as the functional monomer, ethylene glycol dimethacrylate as the crosslinker and toluene or acetone as the porogenic solvent. Thermal polymerization yielded an MIP on which extracts were loaded on a polypropylene SPE cartridge containing the MIP. Analysis of the eluents by HPLC-UV showed high recoveries for vinblastine (93.8\%) and catharanthine (101\%) 
and capacities of 750 and $818 \mu \mathrm{g} / \mathrm{g}$, respectively, whereas for vindoline, recovery was only $33 \%$. The MIP cavity apparently specifically binds catharanthine as a monomer as well as this moiety as part of the dimeric alkaloids.

\subsection{Supercritical $\mathrm{CO}_{2}$}

Supercritical fluid extraction (SFE) has been applied to the isolation of natural products since the late 1970s to a few number of cases like the decafeination of coffee beans and tea leaves (Kaiser, Rompp, \& Schmidt, 2001), but since the early 2000s, this technique has experienced an enormous development particularly in the food, toxicological, pharmaceutical and environmental areas. Compared with conventional organic solvents, supercritical fluids, and particularly $\mathrm{CO}_{2}$, are non-toxic, low cost, environmentally friendly and because of high diffusivity reduce mass-transfer rates. They can be compressed at constant temperature, and therefore, increasing in density and solvent capacity. Their low surface tension facilitates analyte extraction, and since it is gaseous at room temperature and under constant pressure, it can be easily extracted rendering solvent-free analytes (Herrero, Mendiola, Cifuentes, \& Ibañez, 2010). The main supercritical solvent used is $\mathrm{CO}_{2}$ with its low polarity as its main disadvantage, which can be solved with the addition of a polar modifier (co-solvent), for example, 1-10\% methanol or ethanol to expand its extraction range to include more polar compounds, which in turns will also reduce the analyte-matrix interactions improving their quantitative extraction. This can be done in two ways, either by mixing the modifier to the $\mathrm{CO}_{2}$ flow or by mixing it with the raw material in the extraction cell (Mendiola, Herrero, Cifuentes, \& Ibañez, 2007).

Studies regarding SFE have been performed for the major TIA's from Catharanthus, Tabernaemontana and Evodia species (Table 9.1). Lee et al. (1992) extracted vindoline and catharanthine from the leaves of C. roseus without the addition of a modifier. About $67.2 \%(\mathrm{w} / \mathrm{w})$ of vindoline was recovered using a $\mathrm{CO}_{2}$ flow rate of $150 \mathrm{ml} / \mathrm{min}$ at $40{ }^{\circ} \mathrm{C}$, whereas in the case of catharanthine, a higher flow rate of $400 \mathrm{ml} / \mathrm{min}$ at the same temperature had a yield of $52 \%(\mathrm{w} / \mathrm{w})$. Song et al. (1992) compared the addition or absence of ethanol as the modifier for vindoline extraction. The largest amount (58 wt \%) was obtained without the addition of the modifier showing that vindoline solubility is more sensitive to pressure than to temperature. However, a mixture of $\mathrm{CO}_{2}$-methanol-triethylamine (80:12:8) proved to be more effective than methanolic extraction of vinblastine and 
Table 9.1 Experimental conditions for supercritical fluid extraction of TIAs

\begin{tabular}{|c|c|c|c|c|c|c|}
\hline Plant material & Matrix & Target compound & Aim & $\begin{array}{l}\text { Extraction } \\
\text { conditions }\end{array}$ & $\begin{array}{l}\text { Analytical } \\
\text { method }\end{array}$ & References \\
\hline \multirow[t]{4}{*}{$\begin{array}{l}\text { Catharanthus } \\
\text { roseus }\end{array}$} & Leaves & Vindoline, catharanthine & $\begin{array}{l}\text { To selectively extract } \\
\text { vindoline }\end{array}$ & $\begin{array}{l}\mathrm{CO}_{2}, 150 \mathrm{bar} \\
40^{\circ} \mathrm{C}, 10 \mathrm{~h}\end{array}$ & $\begin{array}{l}\text { HPLC, } \\
\text { LC-MS }\end{array}$ & $\begin{array}{l}\text { Lee et al. } \\
(1992)\end{array}$ \\
\hline & Leaves & Vindoline & $\begin{array}{l}\text { To compare extraction } \\
\text { with and without } \\
\text { co-solvent }\end{array}$ & $\begin{array}{l}\mathrm{CO}_{2}+3 \% \\
\text { ethanol, } 300 \text { bar, } \\
35^{\circ} \mathrm{C}, 5 \mathrm{~h}\end{array}$ & $\begin{array}{l}\text { HPLC, } \\
\text { LC-MS }\end{array}$ & $\begin{array}{l}\text { Song et al. } \\
(1992)\end{array}$ \\
\hline & $\begin{array}{l}\text { Aerial } \\
\text { parts } \\
\text { and } \\
\text { roots }\end{array}$ & Vinblastine, vincristine & $\begin{array}{l}\text { To apply a basified SFE } \\
\text { solvent }\end{array}$ & $\begin{array}{l}\mathrm{CO}_{2}-\text { methanol- } \\
\text { triethylamine } \\
(80: 12: 8), 340 \mathrm{bar}, \\
80^{\circ} \mathrm{C}\end{array}$ & LC-MS & $\begin{array}{l}\text { Choi et al. } \\
(2002)\end{array}$ \\
\hline & Leaves & $\begin{array}{l}\text { Catharanthine, vindoline, } \\
3^{\prime}, 4^{\prime} \text {-anhydrovinblastine }\end{array}$ & $\begin{array}{l}\text { To optimize the } \\
\text { method and to compare } \\
\text { it to conventional } \\
\text { extraction methods }\end{array}$ & $\begin{array}{l}\mathrm{CO}_{2}+6.6 \% \\
\text { methanol, } \\
200-300 \mathrm{bar}, \\
80^{\circ} \mathrm{C}, 40 \mathrm{~min}\end{array}$ & $\begin{array}{l}\text { HPLC, } \\
\text { LC-MS }\end{array}$ & $\begin{array}{l}\text { Verma et al. } \\
(2008)\end{array}$ \\
\hline Evodia rutaecarpa & Fruits & Evodiamine, rutaecarpine & $\begin{array}{l}\text { To test the use of } \\
\text { methanol as co-solvent }\end{array}$ & $\begin{array}{l}\mathrm{CO}_{2}+50 \% \\
\text { methanol, } 280 \mathrm{bar} \\
62{ }^{\circ} \mathrm{C}, 1.3 \mathrm{~h}\end{array}$ & HPLC & $\begin{array}{l}\text { Liu et al. } \\
(2010)\end{array}$ \\
\hline $\begin{array}{l}\text { Tabernaemontana } \\
\text { catharinensis }\end{array}$ & $\begin{array}{l}\text { Aerial } \\
\text { parts }\end{array}$ & $\begin{array}{l}\text { Coronaridine, voacangine, } \\
\text { voacristine, voacangine } \\
\text { hydroxylindolenine, } \\
\text { voacristine hydroxylindolenine, } \\
\text { 3-hydroxylcoronaridine }\end{array}$ & $\begin{array}{l}\text { To evaluate } \\
\text { temperature, pressure } \\
\text { and co-solvent }\end{array}$ & $\begin{array}{l}\mathrm{CO}_{2}+4.6 \% \\
\text { ethanol, } 250 \text { bar, } \\
45^{\circ} \mathrm{C}, 2 \mathrm{~h}\end{array}$ & $\begin{array}{l}\text { GC-MS, } \\
\text { GC-FID, } \\
{ }^{1} \mathrm{H} \text { and } \\
{ }^{13} \mathrm{C} \\
\text { NMR }\end{array}$ & $\begin{array}{l}\text { Pereira et al. } \\
(2004)\end{array}$ \\
\hline Uncaria tomentosa & $\begin{array}{l}\text { Root } \\
\text { bark }\end{array}$ & $\begin{array}{l}\text { Isopteropodine, pteropodine, } \\
\text { isomitraphylline, uncarine F, } \\
\text { mitraphylline, speciophylline, } \\
\text { rhynchophylline, } \\
\text { isorhynchophylline }\end{array}$ & $\begin{array}{l}\text { To compare extraction } \\
\text { with and without } \\
\text { co-solvent }\end{array}$ & $\begin{array}{l}\mathrm{CO}_{2}+10 \% \\
\text { methanol, } 253 \mathrm{bar} \\
60{ }^{\circ} \mathrm{C}, 30 \mathrm{~min}\end{array}$ & $\begin{array}{l}\text { HPLC- } \\
\text { MS and } \\
\text { GC-MS }\end{array}$ & $\begin{array}{l}\text { Lopez-Avila, } \\
\text { Benedicto, } \\
\text { and Robaugh } \\
(1997)\end{array}$ \\
\hline
\end{tabular}


vincristine (Choi, Yoo, \& Kim, 2002) and $\mathrm{CO}_{2}$ with $6.6 \%$ methanol for catharanthine (Verma, Hartonen, \& Riekkola, 2008).

Alkaloids from Tabernaemontana catharinensis were extracted using a mixture of supercritical $\mathrm{CO}_{2}$ and $4.6 \%$ ethanol (Pereira et al., 2004). The flow of $\mathrm{CO}_{2}$ was held at $4 \mathrm{bar} / \mathrm{min}$ with a depressurization step of $70 \mathrm{bar}(12 \mathrm{bar} /$ min). Liu, Guo, Chang, Jiang, and Wang (2010) extracted the main alkaloids from unripe fruits of E. rutaecarpa using 50\% methanol as the modifier in a flow rate of $0.4 \mathrm{ml} / \mathrm{min}$ with a static extraction for $5 \mathrm{~min}$ and then dynamic up to $90 \mathrm{~min}$.

\section{ANALYTICAL METHODS}

\subsection{HPLC and hyphenated techniques}

HPLC has been the method of choice for the analysis of alkaloids. It has been recognized since the 1970 s as the most versatile and most widely applied technique for efficient separation and analysis of alkaloids (Verpoorte \& Baerheim Svendsen, 1984). Most of the separations are done on reversedphase materials such as $\mathrm{C}_{8}, \mathrm{C}_{18}$ and phenyl-bonded phases on silica. McCalley (2002) extensively described the importance of the characteristics of the stationary phases for the separation of alkaloids. The most common eluents for the separation of alkaloids are methanol-water and acetonitrile-water buffered at $\mathrm{pH} 2-4$ (buffer strength $>25 \mathrm{mM}$ ) in order to keep alkaloids in their more polar protonated form to reduce tailing due to interaction of the basic nitrogen with the residual acidic silanol groups of the stationary phase (Kingston, 1979; Verpoorte \& Baerheim Svendsen, 1984). Ion-pair chromatography with, for example, long alkyl chain sulfonic acids is also used in alkaloid separations. Various amines such as triethylamine are sometimes added to reduce tailing. For detection, UV absorption is the most widely used since indole alkaloids have strong and specific UV chromophores that can be easily used to identify them, for example, using HPLC with photodiode array detection (DAD). Nevertheless, MS has been a major tool in the identification and structure elucidation of alkaloids, as it not only allows determination of the chemical structure of known and unknown compounds but also offers high sensitivity, and hence the combination of DAD and mass spectroscopy coupled with liquid chromatography, liquid chromatography coupled to mass spectroscopy (LC-MS) is the most selective detection for alkaloids (Verpoorte \& Niessen, 1994). Mobile phases for LC-MS systems using isocratic separations on reversed-phase silica gel type stationary phases are fully volatile acidic eluents containing e.g. formic acid, 
acetic acid, trifluoroacetic acid, ammonium carbonate or ammonium formate (Table 9.2).

Typically, mass spectroscopy data for TIA analysis are acquired in the positive mode and based on the combination of retention time, UV and mass spectra, known compounds are rapidly dereplicated and new structures are identified from different plant matrices such as crude extracts from intact plants and organs, hairy roots, cell suspension cultures and from biological matrices. In multistage MS detection experiments, the use of deuterium-labelled internal standards has proved to be sensitive enough for the accurate quantification of yohimbine from commercially available aphrodisiacs and bark from Pausinystalia yohimbe by using yohimbine- $d_{3}$ (Zanolari, Ndjoko, Ioset, Marston, \& Hostettmann, 2003) or clonazepam $-d_{4}$ for ibogaine and noribogaine determination from human tissues (Chèze, Lenoan, Deveaux, \& Pépin, 2008). Recent approaches using directinjection electrospray ionization (ESI)-MS/MS (Chen, Zhang, Zhang, Chen, \& Chen, 2013; Zhou, Tai, Sun, \& Pan, 2005) or flow-injection ESIMS/MS (Favretto, Piovan, Filippini, \& Caniato, 2001) can omit the sample preparation step and then be used to confirm the presence of alkaloids in different matrices. In addition, these techniques can provide characteristic structural information such as precursor and product ion information, which is useful for multicomponent screening purposes.

\subsection{Ultra high-pressure LC-MS}

One of the latest developments in LC-MS has been the introduction of very $\mathrm{pH}$-stable stationary phases, sub-2- $\mu \mathrm{m}$ particles and monolith columns. This requires high pressures ( $>400$ bar) and is now known as ultra high-pressure liquid chromatography (UHPLC). May achieve up to 100,000 number of plates per time unit $\left(N / t_{0}\right)$ and peak capacities of 900 and reducing the analysis time by a factor of 20 . With the same column length, a three-fold efficiency improvement can be observed compared to $5 \mu \mathrm{m}$ supports (Nguyen, Guillarme, Rudaz, \& Veuthey, 2006).

UHPLC interfaces with high-resolution tandem mass spectrometers and NMR can greatly improve analysis in terms of resolution, speed, reproducibility, sensitivity and unequivocal identification of trace compounds providing confirmative information for studies in e.g. quality control, fingerprinting, authentication, standardization or identification of biomarkers. Other approaches include microfractionation bioactivity-based analysis (Hou et al., 2012), chromatographic profiling ( $\mathrm{Xu}$ et al., 2012), monitoring alkaloid production in cell suspension cultures (He, Yang, Tan, Zhao, \& Hu, 2011; 
Table 9.2 LC systems for quantitative and qualitative analysis of alkaloids

LC conditions column/

particle size $(\mu \mathrm{m}) / \mathrm{mobile}$

phase $A$ and $B /$ internal Analytical

\begin{tabular}{|c|c|c|c|c|c|}
\hline Plant species & Target compound(s) & Matrix & standard (IS) & method & References \\
\hline \multirow[t]{4}{*}{ Catharanthus roseus } & Profiling approach & Roots & $\begin{array}{l}\text { Luna } \mathrm{C}_{18} / 5 / \mathrm{A}: \mathrm{ACN} \text { and } \\
\mathrm{B}: 1 \% \text { acetic acid in water/ } \\
\text { No IS }\end{array}$ & $\begin{array}{l}\text { HPLC- } \\
\text { DAD-ESI- } \\
\text { MS/MS }\end{array}$ & Ferreres et al. (2010) \\
\hline & $\begin{array}{l}\text { Vindoline, vindolidine, } \\
\text { vincristine, vinblastine, } \\
\text { catharanthine, } 19 S- \\
\text { vindolinine, vindolinine }\end{array}$ & $\begin{array}{l}\text { Commercial } \\
\text { extract }\end{array}$ & $\begin{array}{l}\text { Zorbax Eclipse } \mathrm{XDB}_{-} \mathrm{C}_{8} / \\
\text { 5/A: } 0.1 \% \text { triethylamine } \\
\text { and B: methanol/No IS }\end{array}$ & $\begin{array}{l}\text { HPLC-ESI- } \\
\mathrm{MS} / \mathrm{MS}\end{array}$ & Zhou et al. (2005) \\
\hline & Vincristine & $\begin{array}{l}\text { Human } \\
\text { plasma }\end{array}$ & $\begin{array}{l}\text { Luna } \mathrm{C}_{8} / 3 / \mathrm{A}: 1 \% \text { acetic } \\
\text { acid in water and } \mathrm{B} \text { : } \\
\mathrm{ACN} / \text { vincristine and } \\
\text { vinblastine }\end{array}$ & LC-MS/MS & $\begin{array}{l}\text { Guilhaumou et al. } \\
\text { (2010) }\end{array}$ \\
\hline & $\begin{array}{l}\text { Vinblastine, vindoline, } \\
\text { ajmalicine, catharanthine, } \\
\text { vinleurosine }\end{array}$ & Stems & $\begin{array}{l}\mathrm{DL} \mathrm{C}_{18} / 5 / \mathrm{A}: \mathrm{ACN} \text { and B: } \\
10 \mathrm{mM} \text { ammonium } \\
\text { acetate/No IS }\end{array}$ & LC-MS/MS & Chen et al. (2013) \\
\hline Claviceps sp. & $\begin{array}{l}\text { Ergometrine, ergosine, } \\
\text { ergotamine, ergocornine, } \\
\text { ergocryptine, ergocristine }\end{array}$ & $\begin{array}{l}\text { Cereal and } \\
\text { cereal } \\
\text { products }\end{array}$ & $\begin{array}{l}\text { XBridge } \mathrm{MS}_{18} / 3.5 / \mathrm{A} \text { : } \\
\text { water- }-2.2 \mathrm{M} \text { ammonium } \\
\text { bicarbonate-methanol } \\
(85: 5: 10 \mathrm{v} / \mathrm{v} / \mathrm{v}) \text { and } \mathrm{B} \text { : } \\
\text { water }-0.2 \mathrm{M} \text { ammonium } \\
\text { bicarbonate-methanol } \\
(5: 5: 90 \mathrm{v} / \mathrm{v} / \mathrm{v}) / \\
\text { methylergometrine and } \\
\text { dihydroergotamine }\end{array}$ & LC-MS/MS & $\begin{array}{l}\text { Di Mavungu et al. } \\
\text { (2012) }\end{array}$ \\
\hline
\end{tabular}




\begin{tabular}{|c|c|c|c|c|c|}
\hline Evodiae fructus & $\begin{array}{l}\text { Evodiamine, } \\
\text { rutaecarpine }\end{array}$ & $\begin{array}{l}\text { Human } \\
\text { serum }\end{array}$ & $\begin{array}{l}\text { Venusil } \mathrm{XBP} \mathrm{C}_{18} / 5 / \mathrm{A} \text { : } \\
5 \mathrm{mM} \text { ammonium } \\
\text { formate-methanol-water } \\
(85: 15 \mathrm{v} / \mathrm{v}) / \text { evodiamine } \\
\text { and rutaecarpine }\end{array}$ & $\begin{array}{l}\text { LC-ESI- } \\
\text { MS/MS and } \\
\text { LC-APCI- } \\
\text { MS/MS }\end{array}$ & $\begin{array}{l}\text { Wen, Li, Liu, Liao, } \\
\text { and Liu (2006) }\end{array}$ \\
\hline Mitragyna inermis & Uncarine D & Leaves & $\begin{array}{l}\text { Waters } \mathrm{C}_{18} \text { Symmetry/5/ } \\
\mathrm{A} \text { : phosphate-methanol } \\
\text { and B: methanol/ } \\
\text { naphthalene }\end{array}$ & HPLC-DAD & Fiot et al. (2005) \\
\hline M. speciosa & Mitragynine & Urine & $\begin{array}{l}\text { Zorbax } \mathrm{C}_{18} / 5 / \mathrm{A}: 10 \mathrm{mM} \\
\text { ammonium formate in } \\
\text { water and B: } 0.1 \% \text { acetic } \\
\text { acid in ACN/No IS }\end{array}$ & LC-IT-MS & Philipp et al. (2009) \\
\hline Pausinystalia yohimbe & Yohimbine & $\begin{array}{l}\text { Bark and } \\
\text { commercial } \\
\text { aphrodisiacs }\end{array}$ & $\begin{array}{l}\text { Nucleosil } 100-5 \mathrm{C}_{18} \mathrm{AB} / \\
5 / \mathrm{A}: 2 \mathrm{mM} \text { triethylamine } \\
\text { in water and } \mathrm{B}: 2 \mathrm{mM} \text { in } \\
\text { ACN/Yohimbine- } d_{3} \text { (for } \\
\mathrm{MS} \text { ) and codeine (for UV) }\end{array}$ & $\begin{array}{l}\text { HPLC-UV; } \\
\text { HPLC- } \\
\text { APCI-MS; } \\
\text { HPLC-ESI- } \\
\text { MS }\end{array}$ & Zanolari et al. (2003) \\
\hline Rauvolfia serpentina & $\begin{array}{l}\text { Reserpine, ajmaline, } \\
\text { ajmalicine }\end{array}$ & Roots & $\begin{array}{l}\text { Chromolith Performance } \\
\mathrm{C}_{18} / 4.6 / \mathrm{A}: 0.01 \mathrm{M} \\
\text { sodium phosphate and B: } \\
0.5 \% \text { acetic acid in ACN/ } \\
\text { reserpine, ajmaline and } \\
\text { ajmalicine }\end{array}$ & HPLC-DAD & $\begin{array}{l}\text { Srivastava, Tripathi, } \\
\text { Pandey, Verma, and } \\
\text { Gupta (2006) }\end{array}$ \\
\hline
\end{tabular}


Table 9.2 LC systems for quantitative and qualitative analysis of alkaloids-cont'd

\begin{tabular}{|c|c|c|c|c|c|}
\hline Plant species & Target compound(s) & Matrix & $\begin{array}{l}\text { LC conditions column/ } \\
\text { particle size }(\mu \mathrm{m}) / \mathrm{mobile} \\
\text { phase } A \text { and } B / \text { internal } \\
\text { standard (IS) }\end{array}$ & $\begin{array}{l}\text { Analytical } \\
\text { method }\end{array}$ & References \\
\hline $\begin{array}{l}R \text {. serpentina } \times \text { Rhazya } \\
\text { stricta }\end{array}$ & Screening approach & $\begin{array}{l}\text { Hybrid cell } \\
\text { cultures }\end{array}$ & $\begin{array}{l}\text { Nucleosil 100-5 } \mathrm{C}_{18} / \mathrm{A} \text { : } \\
39 \mathrm{mM} \text { sodium phosphate } \\
\text { in ACN and } \mathrm{B}: 3 \mathrm{mM} \\
\text { sodium phosphate- } \\
2.5 \mathrm{mM} \text { hexanesulfonic } \\
\text { acid in ACN/No IS }\end{array}$ & HPLC & Stöckigt et al. (2002) \\
\hline R. verticillata & Fingerprint analysis & $\begin{array}{l}\text { Roots and } \\
\text { rhizomes }\end{array}$ & $\begin{array}{l}\text { Diamonsil } \mathrm{C}_{18} / 5 / \mathrm{A} \text { : } \\
\text { water and } \mathrm{B}: 0.1 \% \text { formic } \\
\text { acid/No IS }\end{array}$ & $\begin{array}{l}\text { LC-Q- } \\
\text { TOF-MS }\end{array}$ & $\begin{array}{l}\text { Hong, Cheng, Wu, } \\
\text { and Zhao }(2010)\end{array}$ \\
\hline \multirow[t]{2}{*}{ Strychnos nux-vomica } & Strychnine & Urine & $\begin{array}{l}\text { Chrompack cyanopropyl/ } \\
\text { 3/A: ACN and B: } 1 \% \\
\text { acetic acid in water/ } \\
\text { nalorphine }\end{array}$ & $\begin{array}{l}\mathrm{LC}- \\
\mathrm{APCI}-\mathrm{MS} / \\
\mathrm{MS}\end{array}$ & $\begin{array}{l}\text { Van Eenoo, } \\
\text { Deventer, Roels, and } \\
\text { Delbeke (2006) }\end{array}$ \\
\hline & & Blood & $\begin{array}{l}\text { Hypurity C18/5/A: ACN } \\
\text { and B: } 20 \mathrm{mM} \text { sodium } \\
\text { dihydrogen phosphate/ } \\
\text { chloroquine }\end{array}$ & LC-DAD & $\begin{array}{l}\text { Duverneuil, de la } \\
\text { Grandmaison, de } \\
\text { Mazancourt, and } \\
\text { Alvarez (2004) }\end{array}$ \\
\hline
\end{tabular}




\begin{tabular}{|c|c|c|c|c|c|}
\hline \multirow[t]{2}{*}{ Tabernanthe iboga } & \multirow[t]{2}{*}{ Ibogaine, noribogaine } & $\begin{array}{l}\text { Human } \\
\text { plasma and } \\
\text { blood }\end{array}$ & $\begin{array}{l}\text { Zorbax eclipse } \mathrm{XD} 8 \mathrm{C}_{8} / \\
5 / \mathrm{A}: 0.02 \% \\
\text { trimethylamine in } \mathrm{ACN} \\
\text { and } \mathrm{B}: 2 \mathrm{mM} \text { ammonium } \\
\text { formate/fluorescein }\end{array}$ & LC-ESI-MS & $\begin{array}{l}\text { Kontrimaviciute, } \\
\text { Breton, Mathieu, } \\
\text { Mathieu-Daudé, and } \\
\text { Bressollee (2006) }\end{array}$ \\
\hline & & $\begin{array}{l}\text { Biological } \\
\text { fluids and } \\
\text { hair }\end{array}$ & $\begin{array}{l}\text { ODB Uptisphere } \mathrm{C}_{18} / 5 / \\
\mathrm{A}: 20 \% \mathrm{ACN} \text { and } \mathrm{B}: \\
2 \mathrm{mM} \text { formate/ } \\
\text { clonazepam- } d_{4}\end{array}$ & $\begin{array}{l}\text { LC-ESI- } \\
\text { MS/MS }\end{array}$ & Chèze et al. (2008) \\
\hline \multirow[t]{2}{*}{ Uncaria tomentosa } & \multirow{2}{*}{$\begin{array}{l}\text { Pteropodine, } \\
\text { isopteropodine, } \\
\text { speciophylline, uncarine, } \\
\text { mytrapylline, } \\
\text { isomytraphylline, } \\
\text { ryncophyllin, } \\
\text { isoryncophyllin, } \\
\text { corynoxeine, } \\
\text { isocorynoxeine }\end{array}$} & $\begin{array}{l}\text { Bark and } \\
\text { leaves }\end{array}$ & $\begin{array}{l}\text { Lichrosorb } \mathrm{C}_{18} / 5 / \mathrm{A} \text { : } \\
30 \mathrm{mM} \text { ammonium } \\
\text { acetate and } \mathrm{B} \text { : methanol- } \\
\text { ACN }(1: 1 \mathrm{v} / \mathrm{v}) / \\
\text { tryptophol }\end{array}$ & $\begin{array}{l}\text { HPLC-ESI- } \\
\text { MS }\end{array}$ & $\begin{array}{l}\text { Montoro, Carbone, } \\
\text { Zuniga-Quiroz, De } \\
\text { Simone, and Pizza } \\
(2004)\end{array}$ \\
\hline & & Bark & $\begin{array}{l}\text { Zorbax XDB } \mathrm{C}_{18} / 5 / \mathrm{A} \text { : } \\
35 \mathrm{mM} \\
\text { triethylammonium acetate } \\
\text { and } \mathrm{B}: \mathrm{ACN} / \\
\text { Mytraphylline }\end{array}$ & $\begin{array}{l}\text { HPLC-ESI- } \\
\text { MS }\end{array}$ & $\begin{array}{l}\text { Bertol, Franco, and } \\
\text { de Oliveira (2012) }\end{array}$ \\
\hline Vinca minor & $\begin{array}{l}\text { Vinblastine, } \\
\text { desacetylvinblastine, } \\
\text { vincristine }\end{array}$ & $\begin{array}{l}\text { Human } \\
\text { plasma }\end{array}$ & $\begin{array}{l}\text { Ultrasphere } \mathrm{C}_{18} / 5 / \mathrm{A} \text { : } \\
15 \mathrm{mM} \text { ammonium } \\
\text { acetate in methanol or } \\
\mathrm{ACN} \text { and } \mathrm{B}: \text { ACN or } \\
\text { methanol/Vinorelbine }\end{array}$ & $\begin{array}{l}\text { LC-APCI- } \\
\text { MS }\end{array}$ & $\begin{array}{l}\text { Ramírez, Ogan, and } \\
\text { Ratainn (1997) }\end{array}$ \\
\hline
\end{tabular}

Abbreviations: ACN, acetonitrile; APCI, atmospheric pressure chemical ionization; ESI, electrospray ionization; FID, flame ionization; IT, ion trap; MS/MS, tandem mass spectroscopy; $\mathrm{Q}$, quadrupole; TOF, time of flight. 
He, Yang, Xiong, et al., 2011), metabonomic approaches (Wang et al., 2010), toxicological studies (Liu, Zhu, Li, Yan, \& Lei, 2011) or in functional studies (Lorenz, Olsovska, Sulc, \& Tudzynski, 2010) using UHPLC-MS where alkaloids are include identified by their retention time, UV spectra, fragmentation pattern data and high-resolution MS data and in some cases confirmed by NMR experiments.

\subsection{Gas chromatography coupled to mass spectroscopy}

Most of the TIAs are polar compounds and not volatile due to their indolyl and tertiary amino group that is not amenable for derivatization, although some of them have been successfully analysed by capillary GC using high temperatures of injection $\left(200-300^{\circ} \mathrm{C}\right)$ and temperature gradients from 100 to $250{ }^{\circ} \mathrm{C}$ (Verpoorte, 2005). The combination of GC with MS is an efficient tool in the preliminary or even complete identification of alkaloids. This approach is used in fingerprinting and bioactivity-guided approaches and even applying hyphenation with capillary electrophoresis (CE) (Table 9.3). For complete identification, fragmentation of the molecular ion is important, which can be achieved by tandem mass spectroscopy $\left(M^{n}\right)$. For quantitative analysis, GC-FID or the specific Nitrogen detector have an advantage over gas chromatography coupled to mass spectroscopy (GC-MS) in the detection and quantitation. In GC-MS, each compound will have a different detector response, which means that absolute quantitation requires calibration curves of each single compound, whereas in the other detection methods, the detector response is more or less similar for all compounds, thus allowing comparison of the peaks within a chromatogram without the need for calibration compounds. That thus allows the analysis of rare alkaloids of which not sufficient material is available for making calibration curves.

Dagnino, Schripsema, Peltenburg, and Verpoorte (1991) showed the feasibility of capillary GC for the analysis of a wide range of TIAs, mainly found in the genus Tabernaemontana. Gallagher et al. (1995) developed a derivatization method to estimate ibogaine levels in biological samples by GC-MS using ibogaine- $d_{3}$ as internal standard. Several derivatizing agents were compared, for example, trifluoroacetyl, heptafluorobutyric anhydride, and trifluoroacetic anhydride in order to determine the best derivatization conditions in terms of choice of chemical reagent, conditions and detection parameters for the reliable quantitation of this alkaloid in different tissues after oral administration. The derivatization of noribogaine and ibogaine by ethylation (Hearn, Pablo, 
Table 9.3 Methods for the detection of alkaloids in different samples using GC-MS techniques

\begin{tabular}{|c|c|c|c|c|c|c|c|}
\hline Compound & Matrix & Work-up & $\begin{array}{l}\text { Internal } \\
\text { standard }\end{array}$ & Derivatization & Stationary phase & $\begin{array}{l}\text { Detection } \\
\text { mode }\end{array}$ & References \\
\hline Ibogaine & Brain & $\begin{array}{l}\text { LLE } \\
\text { (n-hexane) }\end{array}$ & Ibogaine $-d_{3}$ & $\begin{array}{l}\text { Trifluoroacetic } \\
\text { anhydride }\end{array}$ & $\begin{array}{l}\text { DB-5MS } \\
(30 \mathrm{~m} \times 0.25 \mathrm{~mm} \\
0.1 \mu \mathrm{m})\end{array}$ & EI, MS & $\begin{array}{l}\text { Gallagher } \\
\text { et al. } \\
(1995)\end{array}$ \\
\hline \multirow[t]{4}{*}{ Strychnine } & $\begin{array}{l}\text { Liver, lung, } \\
\text { brain, spleen, } \\
\text { skeletal muscle, } \\
\text { bile, urine, blood }\end{array}$ & $\begin{array}{l}\text { LLE (butyl } \\
\text { chloride) }\end{array}$ & Methapyrilene & - & $\begin{array}{l}\text { DB-5MS } \\
(15 \mathrm{~m} \times 0.25 \mathrm{~mm} \\
0.25 \mu \mathrm{m})\end{array}$ & $\begin{array}{l}\text { EI, MS/ } \\
\text { MS, full } \\
\text { scan }\end{array}$ & $\begin{array}{l}\text { Rosano, } \\
\text { Hubbard, } \\
\text { Meola, } \\
\text { and Swift } \\
(2000)\end{array}$ \\
\hline & $\begin{array}{l}\text { Blood, liver, } \\
\text { kidney, small } \\
\text { intestine, urine }\end{array}$ & $\begin{array}{l}\text { LLE } \\
\text { (toluene- } \\
\text { heptane- } \\
\text { isoamyl } \\
\text { alcohol } \\
67: 20: 4 \mathrm{v} / \\
\mathrm{v} / \mathrm{v} \text { ) }\end{array}$ & Papaverine & - & $\begin{array}{l}\text { HP1 } \\
(12.5 \mathrm{~m} \times 0.2 \mathrm{~mm} \\
0.33 \mu \mathrm{m})\end{array}$ & EI, SIM & $\begin{array}{l}\text { Marques } \\
\text { et al. } \\
(2000)\end{array}$ \\
\hline & Blood & SPE & Papaverine & - & $\begin{array}{l}\text { Ultra } 2 \\
(12 \mathrm{~m} \times 0.25 \mathrm{~mm} \\
0.25 \mu \mathrm{m})\end{array}$ & $\begin{array}{l}\text { EI-MS, } \\
\text { SIM }\end{array}$ & $\begin{array}{l}\text { Barroso } \\
\text { et al. } \\
(2005)\end{array}$ \\
\hline & Slimming foods & SPE & $\begin{array}{l}\text { Leucomalachite } \\
\text { green }\end{array}$ & - & $\begin{array}{l}\text { VF-5MS } \\
(30 \mathrm{~m} \times 0.25 \mathrm{~mm} \\
0.25 \mu \mathrm{m})\end{array}$ & $\begin{array}{l}\text { EI-MS/ } \\
\text { MS, full } \\
\text { scan, SIM }\end{array}$ & $\begin{array}{l}\text { Li et al. } \\
(2012)\end{array}$ \\
\hline
\end{tabular}


Table 9.3 Methods for the detection of alkaloids in different samples using GC-MS techniques_cont'd

\begin{tabular}{|c|c|c|c|c|c|c|c|}
\hline Compound & Matrix & Work-up & $\begin{array}{l}\text { Internal } \\
\text { standard }\end{array}$ & Derivatization & Stationary phase & $\begin{array}{l}\text { Detection } \\
\text { mode }\end{array}$ & References \\
\hline $\begin{array}{l}\text { Uleine, } \\
\text { demethoxyaspidormine }\end{array}$ & $\begin{array}{l}\text { Bark of } \\
\text { Himatanthus } \\
\text { lancifolius }\end{array}$ & $\begin{array}{l}\text { SLE }(1 \% \\
\mathrm{HCl})\end{array}$ & - & - & $\begin{array}{l}\mathrm{HP} \\
(30 \mathrm{~m} \times 0.25 \mathrm{~mm} \\
0.25 \mu \mathrm{m})\end{array}$ & MS & $\begin{array}{l}\text { Baggio } \\
\text { et al. } \\
(2005)\end{array}$ \\
\hline Affinisine, voachalotine & $\begin{array}{l}\text { Root bark of } \\
\text { Tabernaemontana } \\
\text { laeta and } \\
\text { T. hystrix }\end{array}$ & SLE & Isatin & - & $\begin{array}{l}\text { DB1 } \\
(30 \mathrm{~m} \times 25 \mathrm{~mm} \\
0.3 \mu \mathrm{m})\end{array}$ & EI, FID & $\begin{array}{l}\text { Vieira } \\
\text { et al. } \\
(2008)\end{array}$ \\
\hline $\begin{array}{l}\text { Aspidospermidine, } \\
\text { demethoxypalosine, } \\
\text { aspidocarpine, } \\
\text { aspidolimine, } \\
\text { fendlerine, } \\
\text { aspidolimidine }\end{array}$ & $\begin{array}{l}\text { Stem bark of } \\
\text { Aspidosperma } \\
\text { spruceaunm }\end{array}$ & $\begin{array}{l}\text { SLE } \\
\text { (methanol) }\end{array}$ & - & - & $\begin{array}{l}\text { DB5-MS } \\
(30 \mathrm{~m} \times 0.25 \mathrm{~mm} \\
0.25 \mu \mathrm{m})\end{array}$ & $\begin{array}{l}\text { EI-MS/ } \\
\text { MS }\end{array}$ & $\begin{array}{l}\text { Aguiar } \\
\text { et al. } \\
(2010)\end{array}$ \\
\hline Yohimbine & $\begin{array}{l}\text { Bark of } \\
\text { Pausinystalia } \\
\text { yohimbe }\end{array}$ & $\begin{array}{l}\text { SLE } \\
\text { (methanol) }\end{array}$ & Diazepam & - & $\begin{array}{l}\text { DB5-MS } \\
(30 \mathrm{~m} \times 0.32 \mathrm{~mm} \\
0.25 \mu \mathrm{m})\end{array}$ & $\begin{array}{l}\text { EI, MS, } \\
\text { SIM }\end{array}$ & $\begin{array}{l}\text { Chen et al. } \\
(2008)\end{array}$ \\
\hline Voacangine, dregamine & $\begin{array}{l}\text { Root extract } \\
\text { from } T . \text { elegans }\end{array}$ & $\begin{array}{l}\text { SLE } \\
\text { (ethanol) }\end{array}$ & - & - & $\begin{array}{l}\text { DB5-MS } \\
(30 \mathrm{~m} \times 32 \mu \mathrm{m} ; \\
0.25 \mathrm{~mm})\end{array}$ & $\begin{array}{l}\text { MS, Scan } \\
\text { mode }\end{array}$ & $\begin{array}{l}\text { Pallant } \\
\text { et al. } \\
(2012)\end{array}$ \\
\hline
\end{tabular}

Abbreviations: EI, electron impact; FID, flame ionization detection; LLE, liquid-liquid extraction; SIM, selected ion monitoring; SLE, solid-liquid extraction. 
Hime, \& Mash, 1995) or silylation (Alburges, Foltz, \& Moody, 1995) after sample clean-up procedures with SPE has also proved to be effective in the determination of these alkaloids from blood, plasma and urine samples.

In bioactivity-guided experiments, a number of alkaloids have been identified from plant extracts or fractions by means of GC-MS (Cardoso, Vilegas, \& Honda, 1998) or GC-FID (Cardoso, Vilegas, \& Pozetti, 1997). Particularly, alkaloids from different Tabernaemontana species (Andrade et al., 2005; Pallant, Cromarty, \& Steenkamp, 2012; Vieira et al., 2008) and Himatanthus lancifolius (Baggio et al., 2005) are amenable for analysis without any derivatization step.

Adulterants such as strychnine along with other compounds present in different commercially available slimming products were determined by GC-MS/MS. Optimization of the method included a sample clean-up step. Since the target compounds are weak bases, the extraction included SPE using a strong cation exchange cartridge which was washed with $2 \%$ formic acid, 30\% methanol-water and 2\% ammoniated methanol (Li et al., 2012).

\subsection{High-speed counter-current chromatography}

High-speed counter-current chromatography (HSCCC) is a two-phase solvent system, without solid phases, instead with liquid stationery phase, to resolve target compounds relying on the different partitioning of solutes between two immiscible solvents which makes it a very effective tool for the preparative separation and purification of natural products (Zhao \& He, 2006).

The preparative isolation of alkaloids can be achieved by means of HSCCC. Because of the ionic nature of alkaloids, systems with a controlled $\mathrm{pH}$ are preferred for their separation. For example, $\mathrm{pH}$-zone-refining counter-current chromatography (CCC) has been quite successful in separating alkaloids based on the $\mathrm{p} K_{\mathrm{a}}$ values, showing the typically characteristic rectangular peaks for the analytes as common in displacement chromatography (Ito \& Ma, 1996). Improved efficiency can be obtained by using ion-pairing gradients, for example, solvent two-phase systems consisting on methanol-chloroform-aqueous phosphate or citrate buffer $(\mathrm{pH} 4)$ containing perchlorate, acetate or chloride as the ion-pairing agent (Fang, Liu, Yang, Wang, \& Huang, 2011; van der Heijden Hermans-Lokkerbol, Verpoorte, \& Baerheim Svendsen, 1987). Important was the observation that ion pairs of alkaloids with chloride and perchlorate are quite well soluble in chloroform-methanol, something which is important information to keep in mind in liquid-liquid partitioning procedures for the isolation of 
alkaloids, as at acidic $\mathrm{pH}$ considerable amounts of alkaloids may pass into an organic solvent due to ion pairing (van der Heijden et al., 1987).

There are few studies on the separation and isolation of TIAs using CCC. They report on the separation of alkaloids from C. roseus (Renault et al., 1999), Strychnos guaianensis (Quetin-Leclercq et al., 1995), S. nux-vomica (Miao Cai, Xiang, An, \& Ito, 1998), Hortia oreadica (Severino et al., 2009), Geissospermum vellosi (Mbeunkui, Grace, \& Lila, 2012) and Tabernaemontana (van der Heijden et al., 1987), and T. catharinensis (Gonçalves, Curcino, Oliveira, \& Braz-Filho, 2011).

Alkaloids from a crude extract of C. roseus as well as an artificial mixture of vinblastine, vincristine and catharanthine were successfully separated as monomers and dimers by means of centrifugal partition chromatography (CPC), a variation of CCC, in the $\mathrm{pH}$-zone refining mode (Renault et al., 1999). The solvent phases used were methyl tert-butyl ether-acetonitrilewater $(4: 1: 5 \mathrm{v} / \mathrm{v} / \mathrm{v})$. The upper organic phase was basified with $8 \mathrm{mM}$ of triethylamine and used as mobile phase (ascending mode) or with $10 \mathrm{mM}$ when used as the stationary phase (descending mode). The lower aqueous phase was acidified with $10 \mathrm{mM} \mathrm{HCl}$ (as a retainer stationary phase) or $8 \mathrm{mM}$ (as a displacer mobile phase).

Quetin-Leclercq et al. (1995) briefly mentioned the fractionation of a chloroform residue by HSCCC when they isolated for the first time guianensine, an alkaloid from the stem bark of Strychnos guianensis using a multilayer-coil separator-extractor and a solvent system of ethyl acetatemethanol-water $(4: 1: 3 \mathrm{v} / \mathrm{v} / \mathrm{v})$ where the lower aqueous phase was used as a stationary phase and the upper organic phase was pumped from the bottom to the upper part of the column which was also applied for strychnine and brucine separation from seeds of $S$. nux-vomica using a two-phase solvent system consisting of chloroform and $0.07 \mathrm{M}$ sodium phosphate in a buffer solution of $0.04 \mathrm{M}$ citric acid (1:1 v/v) (Miao et al., 1998).

Ingkaninan, Hazekamp, Hoek, Balconi, \& Verpoorte (2000), Ingkaninan, Hermans-Lokkerbol, \& Verpoorte (1999), reported the use of CPC for the pre-separation of crude extracts for rapid dereplication of known biological active compounds in plant materials. This included the analysis of several TIAs producing Tabernaemontana plants, allowing, for example, the rapid identification of two active TIAs (tubotaiwine and apparicine).

Severino et al. (2009) demonstrated the advantage of HSCCC in the isolation of the alkaloids rutaecarpin and dictamine from dichloromethane extract of $H$. oreadica leaves. They used the two-phase solvent system composed of $n$-hexane-ethanol-acetonitrile-water (10:8:1:1 v/v/v/v), where 
the upper phase was used as the mobile phase and the lower phase was used as the stationary phase in a tail-to-head elution mode. Further conventional methods of column chromatography yielded rutaecarpin and dictamnine with excellent recoveries compared to the concentration of the compounds quantified simultaneously by LC-APCI-MS/MS analysis of the same extract (93.1\% and $84.9 \%$, respectively). In a similar study, the combination of high-performance counter-current chromatography (HPCCC) and LCMS/MS was successfully established to isolate indole alkaloids from the methanol extract from the stem bark of G. vellosi (Mbeunkui et al., 2012). Extract separation was achieved with the solvent system ethyl acetate-butanol-water $(2: 3: 5 \mathrm{v} / \mathrm{v} / \mathrm{v})$ in an elution-extrusion with the upper phase as stationary phase and the combination of flash column chromatography. Identification of five different indole alkaloids was carried out with ESI multistage mass spectrometry $\left(M^{n}\right)$ data and confirmed by NMR methods.

Voachalotine and 12-methoxy- $N_{\mathrm{b}}$-methylvoachalotine were resolved from the methanolic extract from the roots of $T$. catharinensisin by HSCCC in $4 \mathrm{~h}$ with a solvent system consisting of chloroform-methanol-water (5:10:6 v/v/v) with a $95 \%$ and $97 \%$ purity, respectively, and their identity was confirmed by ${ }^{1} \mathrm{H}$ and ${ }^{13} \mathrm{C}$ NMR experiments (Gonçalves et al., 2011).

Five indole alkaloids from the stem bark of G. vellosii were isolated with a combination of HPCCC and flash chromatography. To further analyse them, ESI-IT-TOF-MS and NMR experiments were conducted (Mbeunkui et al., 2012). In order to study the fragmentation pattern of these alkaloids, multiple tandem mass spectrometric data were produced by CID of the protonated molecule ion based on the most abundant ions $[\mathrm{M}+2 \mathrm{H}]^{2+}$ and $[\mathrm{M}+\mathrm{H}]^{+}$ and a fragmentation pathway geissolosimine, geissospermine, geissoschizoline, geissoschizone and vellosiminol was proposed.

\subsection{Capillary electrophoresis}

$\mathrm{CE}$ represents an attractive analytical technique for the rapid qualitative and quantitative analysis of molecules with a wide range of polarity and molecular weight, including small molecules such as drugs but also macromolecules such as proteins or nucleic acids (Unger, 2009). Because of its versatility and high separation efficiency, CE is an alternative to the widely used RP-HPLC. CE has gained much interest for the analysis of natural products in plant extracts, quality control of herbal medicines, pharmaceutical formulations and food supplements (Ganzera, 2008; Verardo, Gomez-Caravaca, Seura-Carretero, Caboni, \& Fernández-Gutiérrez, 2011). 
The relatively poor sensitivity of CE, resulting from the small loading volumes, can be circumvented by the incorporation of pre-concentration strategies, while the advantages of MS detection are embodied in the improvement of detection sensitivity as well as the capability of both determining the exact mass of analytes and providing structural information, including the possibility to identify and determine co-migrating species in overlapping peaks (Niessen, Tjaden, \& van der Greef, 1993; Ramautar, Somsen, \& de Jong, 2011).

The ideal candidates for CZE are permanently charged molecules such as quaternary alkaloids and electrokinetic chromatography, but in fact, all acidic, basic and neutral compounds can be analysed by CE (Gotti, 2011). For TIA analysis, non-aqueous capillary electrophoresis (NACE) has been the most widely used since electrolytes such as ammonium acetate and ammonium formate can be used, allowing the hyphenation of CE and MS (Scriba, 2007). Buffer systems and CE methods used for TIA analysis are listed in Table 9.4.

NACE was useful for the separation of 11 Vinca alkaloids from an artificial mixture. Results were compared to those of HPLC using UV traces of both methods at $214 \mathrm{~nm}$ showing that although HPLC is more sensitive than $\mathrm{CE}$ in terms of limit of detection (LOD) and limit of quantification (LOQ), CE can be a good alternative by reducing analysis time and giving better resolution (Barthe et al., 2002).

Posch, Martin, et al. (2012) described an NACE-MS method to screen the psychoactive alkaloids present in two commercial preparations from Mitragyna speciosa as a quality control for added active compounds like the opioid O-desmethyltramadol, which can be fatal for humans. The use of a non-aqueous buffer system allowed the separation of diastereomers of mytraginine. The same methodology proved to have a high resolving power for the separation of iboga alkaloids from Voacanga africana although the choice of detector was not enough to discriminate between analytes with similar masses and migration times. In a similar study with preparations from $M$. speciosa, a higher selectivity and resolution were observed when BGE was switched to ammonium formate (Posch, Müller, et al., 2012). For the analysis of indole alkaloids from the root bark of $P$. yohimbe by NACE and GC-MS, the latter proved to be more sensitive (LOD 0.6 and $1.0 \mu \mathrm{g} / \mathrm{ml}$, respectively) in terms of identification (Fig. 9.1; Chen et al., 2008).

An aqueous $\mathrm{CE}$ system using $\alpha, \beta$ or $\gamma$ cyclodextrins (CD) was tested for the enantiomeric separation of vincamine, vinpocetine and vincadifformine. The best separations were achieved with $\beta-C D$ and $\gamma$-CD. The proposed structures for the inclusion complexes were based on rotating-frame nuclear 
Table 9.4 Buffer systems and CE methods for the analysis of terpenoid indole alkaloids from different plant species

\begin{tabular}{|c|c|c|c|c|c|}
\hline Plant species & Target compound & $\begin{array}{l}\text { CE } \\
\text { method }\end{array}$ & Electrolyte & $\begin{array}{l}\text { Analytical } \\
\text { method }\end{array}$ & References \\
\hline \multirow[t]{2}{*}{ Catharanthus roseus } & Vinblastine, vincristine & $\mathrm{CZE}$ & $\begin{array}{l}0.2 \mathrm{M} \text { ammonium } \\
\text { acetate, } \mathrm{pH} 6.2\end{array}$ & UV & $\begin{array}{l}\text { Chu, Bodnar, White, and } \\
\text { Bowman (1996) }\end{array}$ \\
\hline & $\begin{array}{l}\text { Vinblastine, vindoline, } \\
\text { catharanthine }\end{array}$ & $\mathrm{CE}$ & $\begin{array}{l}20 \mathrm{mM} \text { ammonium } \\
\text { acetate in } 1.5 \% \text { acetic } \\
\text { acid }\end{array}$ & MS & $\begin{array}{l}\text { Chen, Li, Zhang, Chen, } \\
\text { and Chen (2011) }\end{array}$ \\
\hline Claviceps purpurea & $\begin{array}{l}\text { Ergonovinine, } \\
\text { ergonovine, ergocornine, } \\
\text { ergocryptine, ergocornine, } \\
\text { ergocristine, ergosine, } \\
\text { ergocristinine, ergotamine }\end{array}$ & $\mathrm{CE}$ & $\begin{array}{l}20 \mathrm{mM} \beta-\mathrm{CD}, 8 \mathrm{mM} \\
\gamma \text {-CD, } 2 \mathrm{M} \text { urea, } 0.3 \% \\
\text { PVA in phosphate } \\
\text { buffer, pH } 2.5\end{array}$ & UV & Frach and Blaschke (1998) \\
\hline Evodiae fructus & $\begin{array}{l}\text { Evodiamine, rutaecarpine, } \\
\text { carboxyevodiamine, } \\
\text { 1-methyl-2-nonyl-4(1H)- } \\
\text { quinolone, } \\
\text { 1-mehtyl-2-[( } Z)-6- \\
\text { undecenyl]-4(1H)- } \\
\text { quinolone, } \\
\text { 1-methyl-2-undecyl-4 } \\
\text { (1H)-quinolone, } \\
\text { evocarpine, } \\
\text { 1-methyl-2-[(6Z,9Z)-6,9- } \\
\text { pentadecadienyl]-4(1H)- } \\
\text { quinolone, } \\
\text { dihydroevodiamine }\end{array}$ & $\begin{array}{l}\text { CZE, } \\
\text { MEKC }\end{array}$ & $\begin{array}{l}\text { CZE: } 40 \mathrm{mM} \text { sodium } \\
\text { dihydrogen phosphate- } \\
\text { ACN }(9: 1 \mathrm{v} / \mathrm{v}) \\
\text { MEKC: } 20 \mathrm{mM} \\
\text { phosphate, } 40 \mathrm{mM} \text { SDS, } \\
9 \mathrm{mM} \text { sodium borate, } \\
\text { pH } 7.31\end{array}$ & UV & $\begin{array}{l}\text { Lee, Chuang, and Sheuu } \\
(1996)\end{array}$ \\
\hline
\end{tabular}


Table 9.4 Buffer systems and CE methods for the analysis of terpenoid indole alkaloids from different plant species-cont'd

\begin{tabular}{|c|c|c|c|c|c|}
\hline Plant species & Target compound & $\begin{array}{l}\text { CE } \\
\text { method }\end{array}$ & Electrolyte & $\begin{array}{l}\text { Analytical } \\
\text { method }\end{array}$ & References \\
\hline \multirow[t]{2}{*}{ Mitragyna speciosa } & $\begin{array}{l}\text { Mitragynine, } \\
\text { paynantheine, } \\
\text { 7-hydroxy-mitragynine }\end{array}$ & NACE & $\begin{array}{l}60 \mathrm{mM} \text { ammonium } \\
\text { formate, } 5 \% \text { acetic } \\
\text { acid in } \mathrm{ACN}\end{array}$ & qTOF-MS & $\begin{array}{l}\text { Posch, Müller, Schulz, } \\
\text { Pütz, and Huhn (2012) }\end{array}$ \\
\hline & $\begin{array}{l}\text { Mitragynine, } \\
\text { speciogynine, } \\
\text { speciociliatine, } \\
\text { mitraciliatine }\end{array}$ & NACE & $\begin{array}{l}58 \mathrm{mM} \text { ammonium } \\
\text { formate, } 1 \mathrm{M} \text { acetic } \\
\text { acid in } \mathrm{ACN}\end{array}$ & qTOF-MS & $\begin{array}{l}\text { Posch, Martin, Pütz, and } \\
\text { Huhn (2012) }\end{array}$ \\
\hline Pausinystalia yohimbe & Yohimbine & NACE & $\begin{array}{l}20 \mathrm{mM} \text { ammonium } \\
\text { acetate in } 0.5 \% \text { acetic } \\
\text { acid }\end{array}$ & UV & Chen et al. (2008) \\
\hline Phellodendron wilsonii & $\begin{array}{l}\text { Berberine, palmatine, } \\
\text { jatrorrhizine, } \\
\text { phellondendrine, } \\
\text { tetrahydropalmatine, } \\
\text { magnoflorine, thalphenine }\end{array}$ & $\mathrm{CE}$ & $\begin{array}{l}60 \mathrm{mM} \text { ammonium } \\
\text { acetate in } 40 \% \\
\text { methanol, } \mathrm{pH} 4.5\end{array}$ & UV, MS & $\begin{array}{l}\text { Henion, Mordehai, and } \\
\text { Cai (1994) }\end{array}$ \\
\hline Psilocybe semilanceata & Psilocybin, baeocystin & $\mathrm{CZE}$ & $\begin{array}{l}10 \mathrm{mM} \text { borate, } 10 \mathrm{mM} \\
\text { phosphate, } 25 \mathrm{mM} \text { SDS, } \\
\text { pH } 11.5\end{array}$ & UV & $\begin{array}{l}\text { Pedersen-Bjergaard, } \\
\text { Rasmussen, and Sannes } \\
(1998)\end{array}$ \\
\hline $\begin{array}{l}\text { Rauvolfia serpentina, } \\
\text { Rauvolfia } \\
\text { serpentina } \times \text { Rhazya stricta, }\end{array}$ & $\begin{array}{l}\text { Gramine, tryptamine, } \\
\text { serpentine, alstonine, } \\
\beta \text {-methylajmaline, }\end{array}$ & $\mathrm{CZE}$ & $\begin{array}{l}100 \mathrm{mM} \text { ammonium } \\
\text { acetate in ACN (1:1 v/ } \\
\mathrm{v}), \mathrm{pH} 3.1\end{array}$ & UV, MS & $\begin{array}{l}\text { Stöckigt et al. (2002), } \\
\text { Stöckigt, Unger, Belder, } \\
\text { and Stöckigtt (1997) and }\end{array}$ \\
\hline
\end{tabular}




\begin{tabular}{|c|c|c|c|c|c|}
\hline $\begin{array}{l}\text { Aspidosperma quebracho- } \\
\text { blanco }\end{array}$ & $\begin{array}{l}\text { tabersonine, vinblastine, } \\
\text { corynanthine, vincristine, } \\
\text { raufloridine, ajmaline, } \\
\text { yohimbic acid, } \\
\text { deserpidine, reserpine, } \\
\text { rescinnamine }\end{array}$ & & & & $\begin{array}{l}\text { Unger, Stöckigt, Belder, } \\
\text { and Stöckigtt (1997) }\end{array}$ \\
\hline \multirow[t]{4}{*}{ Strychnos nux-vomica } & Strychnine, brucine & MEKC & $\begin{array}{l}50 \mathrm{mM} \text { phosphate, } \\
100 \mathrm{mM} \text { SDS in ACN } \\
(4: 1 \mathrm{v} / \mathrm{v}), \mathrm{pH} 2.0\end{array}$ & & $\begin{array}{l}\text { Wang, Han, Wang, Zang, } \\
\text { and Wu (2006) }\end{array}$ \\
\hline & & $\mathrm{CZE}$ & $\begin{array}{l}10 \mathrm{mM} \text { phosphate buffer } \\
\text { in methanol }(9: 1 \mathrm{v} / \mathrm{v}) \text {, } \\
\mathrm{pH} 2.5\end{array}$ & UV & Zong and Che (1995) \\
\hline & & NACE & $\begin{array}{l}25 \mathrm{mM} \text { Tris boric acid, } \\
\text { methanol-ACN }(6: 2 \mathrm{v} / \\
\mathrm{v}), \mathrm{pH} 4.0\end{array}$ & UV & $\begin{array}{l}\mathrm{Gu}, \mathrm{Li}, \mathrm{Zhu} \text {, and Zou } \\
\text { (2006) }\end{array}$ \\
\hline & & & $\begin{array}{l}30 \mathrm{mM} \text { ammonium } \\
\text { acetate, acetic acid- } \\
\text { ACN }(1: 1.5 \mathrm{v} / \mathrm{v}) \text { in } \\
\text { methanol }\end{array}$ & UV & Li et al. (2006) \\
\hline S. pierrian & $\begin{array}{l}\text { Strychnine, brucine, } \\
\text { novacine, icajine }\end{array}$ & & $\begin{array}{l}80 \mathrm{mM} \text { ammonium } \\
\text { acetate, } 0.1 \% \text { acetic acid } \\
\text { in water-methanol } \\
(4: 6 \mathrm{v} / \mathrm{v})\end{array}$ & UV, MS & Feng, Yuan, and Lii (2003) \\
\hline Uncaria tomentosa & Oxindole alkaloids & $\mathrm{CZE}$ & $\begin{array}{l}20 \mathrm{mM} \text { phosphate } \\
\text { buffer, pH } 5.6\end{array}$ & UV & $\begin{array}{l}\text { Stuppner, Sturn, and } \\
\text { Konwalinkaa (1992) }\end{array}$ \\
\hline
\end{tabular}


Table 9.4 Buffer systems and CE methods for the analysis of terpenoid indole alkaloids from different plant species—cont'd

\begin{tabular}{|c|c|c|c|c|c|}
\hline Plant species & Target compound & $\begin{array}{l}\text { CE } \\
\text { method }\end{array}$ & Electrolyte & $\begin{array}{l}\text { Analytical } \\
\text { method }\end{array}$ & References \\
\hline \multirow[t]{2}{*}{ Vinca } & $\begin{array}{l}\text { Catharanthine, } \\
\text { vinorelbine, } \\
\text { anhydrovinblastine, } \\
\text { vinflunine, vindoline, } \\
\text { 4-O-deacetylvinorelbine, } \\
\text { 4-O-deaceylvinflunine, } \\
\text { vindesine, } 4^{\prime} \text { - } \\
\text { deoxy- } 20^{\prime}, 20^{\prime} \text { - } \\
\text { difluorovinblastine, } \\
\text { vincristine }\end{array}$ & NACE & $\begin{array}{l}50 \mathrm{mM} \text { ammonium } \\
\text { acetate, } 25 \mathrm{mM} \text { SDS, } \\
0.6 \mathrm{M} \text { acetic acid in } \\
\text { methanol-ACN } \\
(75: 25 \mathrm{v} / \mathrm{v}), \mathrm{pH} 7.7\end{array}$ & $\begin{array}{l}\text { HPLC- } \\
\text { DAD }\end{array}$ & Barthe et al. (2002) \\
\hline & $\begin{array}{l}\text { Vincamine, vinpocetine, } \\
\text { vincadiformine }\end{array}$ & $\mathrm{CE}-\mathrm{CD}$ & $\begin{array}{l}0.25-50 \mathrm{mM} \text { CD, } \\
15 \mathrm{mM} \mathrm{NaOH}, \mathrm{pH} 2.5\end{array}$ & $\begin{array}{l}\text { UV, } \\
\text { NMR }\end{array}$ & Sohajda et al. (2010) \\
\hline Voacanga africana & $\begin{array}{l}\text { Voacamine, ibogaine, } \\
\text { voacangine, } \\
\text { 3-oxovoacangin }\end{array}$ & NACE & $\begin{array}{l}58 \mathrm{mM} \text { ammonium } \\
\text { formate, } 1 \mathrm{M} \text { acetic } \\
\text { acid in } \mathrm{ACN}\end{array}$ & qTOF-MS & Posch, Martin, et al. (2012) \\
\hline
\end{tabular}

Abbreviations: ACN, acetonitrile; CD, cyclodextrin; CE, capillary electrophoresis; CZE, capillary zone electrophoresis; MEKC, micellar electrokinetic chromatography; NACE, non-aqueous capillary electrophoresis; PVA, polyvinyl alcohol; SDS, sodium dodecyl sulphate. 


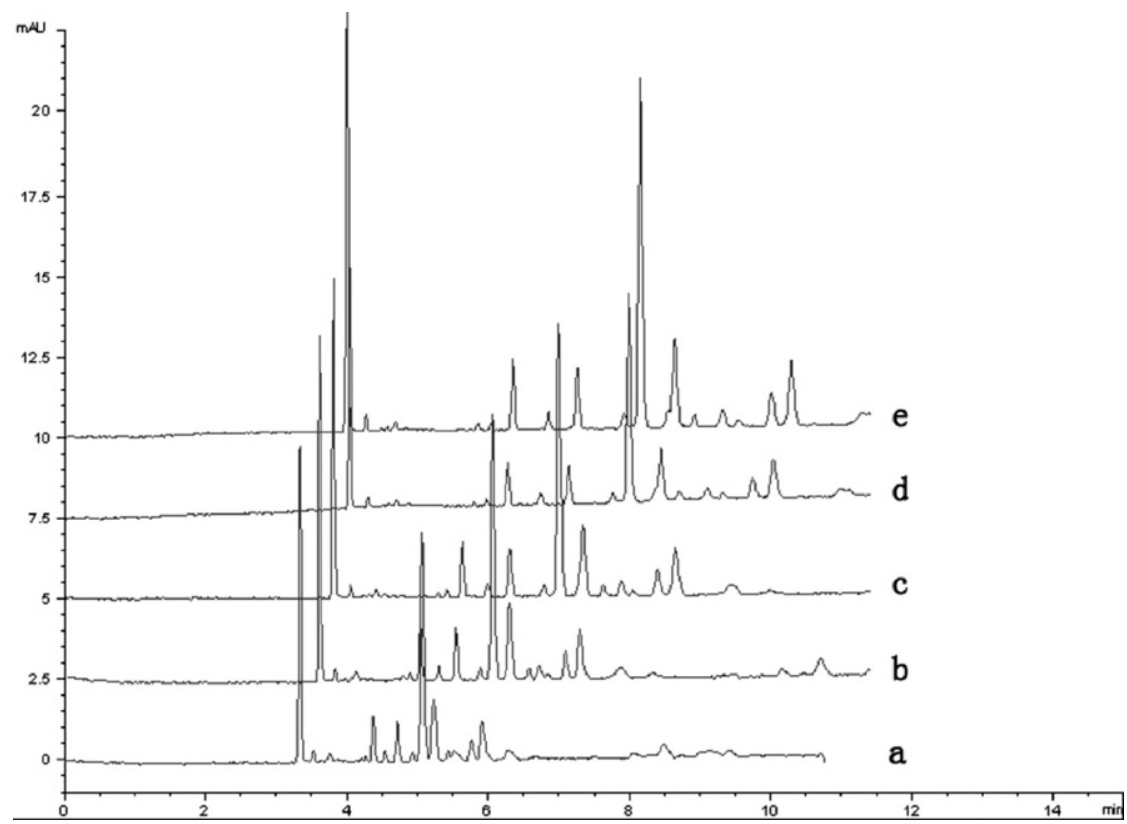

Figure 9.1 Electropherograms of several concentrations of ammonium acetate on the mobility of yohimbe, diazepam and berberine. Buffer solutions (a) $10 \mathrm{mM}$, (b) $15 \mathrm{mM}$, (c) $20 \mathrm{mM}$, (d) $25 \mathrm{mM}$, and (e) $30 \mathrm{mM}$ ammonium acetate in $0.5 \%$ acetic acid. Adapted with permission from Chen et al. (2008).

Overhauser effect correlation spectroscopy (ROESY) experiments and their stability constants were determined by ${ }^{1} \mathrm{H}$ NMR chemical shift titrations for the three alkaloids (Sohajda et al., 2010). Using the same CDs, ergot alkaloids were successfully resolved in 12 min analysis time and 30-fold increased sensitivity when a laser-induced fluorescence detector was used (Frach \& Blaschke, 1998).

\subsection{Quantitative nuclear magnetic resonance}

One of the major advantages of quantitative nuclear magnetic resonance (qNMR) is its primary analytical characteristic, because of which it can be applied in the quantitative estimation of purity of compounds without using any specific reference standard (Lindon \& Nicholson, 2008). NMR-based metabolomics provides absolute and relative quantification of several metabolites in biological samples without separation of individual components in normal or modulated metabolism, so qNMR spectroscopy 
has been widely applied in environmental toxicity, drug toxicity, disease diagnosis, cancer metabolism, pathophysiology of disease, stress, nutrition, drug metabolism, plant metabolism, bacterial metabolism and cell-virus interactions (Bharti \& Roy, 2012).

Camptothecin, 9-methoxycamptothecin, pumiloside and trigonelline were quantified by ${ }^{1} \mathrm{H}$ NMR analysis in root, stems and leaves from Nothapodytes foetida using DMSO- $d_{6}$ as solvent and 3,4,5trimethoxybenzaldehyde as internal standard ( $\mathrm{Li}, \mathrm{Lin}, \& \mathrm{Wu}, 2005)$. The signals of $\mathrm{H}-7, \mathrm{H}-10, \mathrm{H}-19$ and $\mathrm{H}-2$ were selected as target signals for quantification of each alkaloid, respectively. Quantitation data were compared and confirmed to that of HPLC.

\section{APPLICATIONS IN FINGERPRINT ANALYSIS}

\subsection{NMR and LC-MS-based metabolic fingerprinting on TIAs}

NMR spectroscopy has a long-standing tradition to be applied to the characterization of pure compounds as it has been the case for the structural elucidation of TIAs. The continuous development of more and more sophisticated one-dimensional (1D) and 2D pulse sequences in NMR, various structure elucidation strategies have been developed and in the early 2000s, the unambiguous NMR-based structure elucidation of bisindoles is now inconceivable without using a 'holistic' NMR approach, that is, a full ${ }^{1} \mathrm{H}$ and ${ }^{13} \mathrm{C}$ NMR assignment in conjunction with the establishment of all spin-spin connectivities by a broad range of 2D-NMR methods, that is, homonuclear correlated spectroscopy (COSY), total correlated spectroscopy (TOCSY), heteronuclear single quantum coherence (HSQC), heteronuclear multiple bond correlation (HMBC), heteronuclear multiple quantum coherence (HMQC), nuclear Overhauser effect spectroscopy (NOESY) or ROESY experiments (Beni, Hada, Dubrovay, \& Szantay, 2012).

There are excellent reviews covering the structural elucidation of the alkaloids in Catharanthus (Beni et al., 2012; Blaskó \& Cordell, 1990; Dubrovay, Háda, Béni, \& Szántay, 2012; Háda et al., 2012), Aspidosperma (Guimaraes, Braz-Filho, \& Vieira, 2012), Tabernaemontana (Danieli \& Palmisano, 1987; Nielsen, Hazell, Hazell, Ghia, \& Torssell, 1994; Talapatra, Patra, \& Talapatra, 1975), and Strychnos (Penelle et al., 2001, 2000; Rasoanaivo, Martin, Guittet, \& Frappier, 2002) already published. However, the 
description of these experiments is beyond the scope of this discussion and interested readers are advised to consult the aforementioned publications.

Even though NMR is a crucial tool for identification and structure elucidation of pure samples, it can also make important contributions to the metabolic profiling by complementing MS-based approaches (Forseth \& Schroeder, 2011). NMR-based metabolomics can be effectively applied to characterize and distinguish plants on species and genotype levels, different plant tissues within the same plant as well as for the detection of adulterants in foods and in health supplements for quality control (Holmes, Tang, Wang, \& Seger, 2006). NMR can be integrated with chromatography to analyse herbal products to generate standardized 'metabolic fingerprints' which contains markers for activity (Heyman \& Meyer, 2012).

One of the most common adulterants in Strychnos preparations is the 'false angostura bark', Galipea officinalis, whose bark closely resembles that of $S$. nux-vomica. An ${ }^{1} \mathrm{H}$ NMR method was developed for the quantitative analysis of strychnine and brucine in seeds and stem bark from $S$. nuxvomica (Frederich, Choi, \& Verpoorte, 2003) along with a multivariate analysis which was useful for the metabolic profiling of $S$. nux-vomica, S. ignatii and S. icaja (Frederich et al., 2004). With this study, it was possible to discriminate the three species according to the composition in different organs, that is, seeds, leaves, stem bark and root bark. The compounds responsible for this discrimination were strychnine, brucine, loganin, fatty acids, icajine and sungucine. Strychnos nux-vomica and S. icaja stem bark could be distinguished by their content of brucine, but it was not possible to discriminate between the stem from $S$. nux-vomica and its adulterant arguing that the original material must have come from either stem bark or root bark.

Another interesting example concerns NMR-based profiling of Cinchona alkaloids in museum samples dating from 1850 to 1950 in order to determine the variation in contents from 117 different bark samples (Yilmaz, Nyberg, \& Jaroszewski, 2012). An extraction system was developed using chloroform- $d_{1}$, methanol- $d_{4}, \mathrm{D}_{2} \mathrm{O}$ and aqueous $70 \%$ perchloric acid $(5: 5: 1: 1 \mathrm{v} / \mathrm{v} / \mathrm{v} / \mathrm{v})$. With an initial principal component analysis (PCA), it was possible to rule out four mislabelled samples that did not correspond at all to the Cinchona materials. With STOCSY-CA (statistical total correlation spectroscopy component analysis), it was possible to draw the conclusion that the variation methods in extracted alkaloids is not due to decomposition of quinine but an effect of the different cultivation methods of Cinchona trees over time. 
Using 1D and 2D NMR, a comparison between the metabolic profile of healthy and phytoplasma-infected C. roseus plants was conducted along with multivariate data analysis in order to characterize and identify the metabolites responsible for the discrimination of the samples (Choi et al., 2004). Infected leaves showed an increase in signals of $\mathrm{H}-9$ at $\delta 6.89$ corresponding to vindoline showing a twofold increase than in healthy plants. The TIA precursors secologanin and loganic acid as well as chlorogenic acid and sugars, were four times higher than in healthy plants.

An MS-based fingerprint analysis was reported for yohimbe bark, and 18 different commercial dietary supplements, in order to determine the presence of yohimbine in the samples as well as to assess the quality of these supplements in the form of tablet, capsule or liquid (Sun \& Chen, 2012). MS data were only used to confirm the identities of yohimbine, corynanthine and some other alkaloids, but the fingerprint analysis was conducted using the characteristic peaks in a chromatographic approach. In this case, all peaks are normalized against the area of yohimbine. With this method, the authors unambiguously demonstrated that 10 of the tested commercial preparations did not contain the amount of yohimbine claimed in the label of the product.

Mass spectroscopy does not only give the molecular weight, but each compound has also a characteristic fragmentation pattern, which is very useful for identification in GC-MS and LC-MS. Hesse (1974) has brought together all the information on mass spectroscopy of indole alkaloids, a very useful tool for identifying the identity of indole alkaloids.

\section{CONCLUSIONS}

The analysis of TIAs is a challenging task because of their complex chemical structures, usual low abundance and their difficult and timeconsuming extraction procedures from different plant materials as well as from biological fluids. Only highly selective and sensitive methods will be suitable for such analyses. Sensitivity is the disadvantage of ${ }^{1} \mathrm{H}$ NMR when it comes to low contents of alkaloids in plant extracts, yet it is the only technique, which produces signals directly correlated with the amount of analytes in the sample. Even though CE offers some potential improvements in TIAs separation, it often faces sensitivity problems and in the case of GC, although a powerful tool, it is only suitable for a limited number of alkaloids which are volatile or amenable for derivatization. Consequently, liquid chromatographic or electrophoretic techniques in combination with 
different detectors have been mostly employed for TIAs analysis. Thus, HPLC in the reversed-phase mode has been and is the preferred separation technique for the analysis of TIAs.

The UV and DAD detection are robust detectors for targeted analysis. LC-MS offers further resolution and are of interest for more in depth analyses. Metabolomics as a novel approach is based on the different methods discussed here. But it requires a strict standardization to be able to store the results of the analyses with other laboratories. It should thus be based on standard protocols and public databases where the data are stored. Considering the extensive data presented in this review, it is clear that there is still a very long way to go to come to a chromatography based metabolomics in which all alkaloids can be analyzed. NMR despite its disadvantages of not being sensitive enough, seems closest to become a metabolomic platform in which also alkaloids can be analyzed.

\section{REFERENCES}

Aguiar, G. P., Wakabayashi, K. A. L., Luz, G. F., Oliveira, V. B., Mathias, L., Vieira, I. J. C., et al. (2010). Fragmentation of plumeran indole alkaloids from Aspidosperma spruceanum by electrospray ionization tandem mass spectrometry. Rapid Communications in Mass Spectrometry, 24, 295-308.

Alburges, M. E., Foltz, R. L., \& Moody, D. E. (1995). Determination of ibogaine and 12-hydroxy-ibogamine in plasma by gas chromatography-positive ion chemical ionization mass spectrometry. Journal of Analytical Toxicology, 19, 381-386.

Andrade, M. T., Lima, J. A., Pinto, A. C., Rezende, C. M., Carvalho, M. P., \& Epifanio, R. A. (2005). Indole alkaloids from Tabernaemontana australis (Müell. Arg) Miers that inhibit acetylcholinesterase enzyme. Bioorganic \& Medicinal Chemistry, 13, 4092-4095.

Baerheim Svendsen, A., \& Verpoorte, R. (Eds.), (1983). Chromatography of alkaloids, part I, thin-layer chromatography. In Chromatography library, Vol. 23A, (pp. 1-534). Amsterdam: Elsevier.

Baggio, C. H., de Martini, G., de Souza, W., de Moraes, C. A., Brandao, L. M., Rieck, L., et al. (2005). Gastroprotective mechanisms of indole alkaloids from Himatanthus lancifolius. Planta Medica, 71, 733-738.

Barroso, M., Gallardo, E., Margalho, C., Marques, E., Vieira, D. N., \& López-Rivadulla, M. (2005). Determination of strychnine in human blood using solid-phase extraction and GC-EI-MS. Journal of Analytical Toxicology, 29, 383-386.

Barthe, L., Ribet, J. P., Pelissou, M., Degude, M. J., Fahy, J., \& Duflos, A. (2002). Optimization of the separation of Vinca alkaloids by nonaqueous capillary electrophoresis. Journal of Chromatography. A, 968, 241-250.

Beni, Z., Hada, V., Dubrovay, Z., \& Szantay, C., Jr. (2012). Structure elucidation of indoleindoline type alkaloids: A retrospective account from the point of view of current NMR and MS technology. Journal of Pharmaceutical and Biomedical Analysis, 69, 106-124.

Bertol, G., Franco, L., \& de Oliveira, B. H. (2012). HPLC analysis of oxindole alkaloids in Uncaria tomentosa: Sample preparation and analysis optimization by factorial design. Phytochemical Analysis, 23, 143-151.

Bharti, S. K., \& Roy, R. (2012). Quantitative ${ }^{1}$ H NMR spectroscopy. Trends in Analytical Chemistry, 35, 5-26. 
Blaskó, G., \& Cordell, G. A. (1990). Isolation, structure elucidation and biosynthesis of the bisindole alkaloids of Catharanthus. In B. Arnold, \& S. Matthew (Eds.), The alkaloids: Chemistry and pharmacology (pp. 1-76). Orlando, FL: Academic Press.

Cao, M., Muganga, R., Nistor, I., Tits, M., Angenot, L., \& Frederich, M. (2012). LC-SPENMR-MS analysis of Strychnos usambarensis fruits from Rwanda. Phytochemistry Letters, 5 , $170-173$.

Cardoso, C. A. L., Vilegas, W., \& Honda, N. K. (1998). Qualitative determination of indole alkaloids, triterpenoids and steroids of Tabernaemontana hilariana. Journal of Chromatography. A, 808, 264-268.

Cardoso, C. A. L., Vilegas, W., \& Pozetti, G. L. (1997). Gas chromatography analysis of indole alkaloids from Tabernaemontana hilariana. Journal of Chromatography. A, 788, 204-206.

Chen, Q., Li, N., Zhang, W., Chen, J., \& Chen, Z. (2011). Simultaneous determination of vinblastine and its monomeric precursors vindoline and catharanthine in Catharanthus roseus by capillary electrophoresis-mass spectrometry. Electrophoresis, 34, 2885-2892.

Chen, Q., Li, P., Zhang, Z., Li, K., Liu, J., \& Li, Q. (2008). Analysis of yohimbine alkaloid from Pausinystalia yohimbe by non-aqueous capillary electrophoresis and gas chromatography-mass spectrometry. Journal of Separation Science, 31, 2211-2218.

Chen, Q., Zhang, W., Zhang, Y., Chen, J., \& Chen, Z. (2013). Identification and quantification of active alkaloids in Catharanthus roseus by liquid chromatography-ion trap mass spectrometry. Food Chemistry, 139, 845-852.

Chèze, M., Lenoan, A., Deveaux, M., \& Pépin, G. (2008). Determination of ibogaine and noribogaine in biological fluids and hair by LC-MS/MS after Tabernanthe iboga abuse. Iboga alkaloids distribution in a drowning death case. Forensic Science International, 176, 58-66.

Choi, Y. H., Tapias, E. C., Kim, H. K., Lefeber, A. W. M., Erkelens, C., Verhoeven, J. T. J., et al. (2004). Metabolic discrimination of Catharanthus roseus leaves infected by phytoplasma using ${ }^{1} \mathrm{H}-\mathrm{NMR}$ spectroscopy and multivariate data analysis. Plant Physiology, 135, 2398-2410.

Choi, Y. H., Yoo, K. P., \& Kim, J. (2002). Supercritical fluid extraction and liquid chromatography-electrospray mass analysis of vinblastine from Catharanthus roseus. Chemical and Pharmaceutical Bulletin, 50, 1294-1296.

Chu, I., Bodnar, J. A., White, E. L., \& Bowman, R. N. (1996). Quantification of vincristine and vinblastine in Catharanthus roseus plants by capillary zone electrophoresis. Journal of Chromatography. A, 755, 281-288.

Dagnino, D., Schripsema, J., Peltenburg, A., \& Verpoorte, R. (1991). Capillary gas chromatographic analysis of indole alkaloids: Investigation of the indole alkaloids present in Tabernaemontana divaricata cell suspension culture. Journal of Natural Products, 54, 1558-1563.

Danieli, B., \& Palmisano, G. (1987). Alkaloids from Tabernaemontana. In A. Brossi (Ed.), The alkaloids: Chemistry and pharmacology (pp. 1-130). New York: Elsevier Inc.

de Castro, A., Perez, T., de Carvalho, G. M., Pinto, L. L., da Silva, C. C., Tanaka, J. C., et al. (2012). Anti-leishmanial activity of alkaloid extracts obtained from different organs of Aspidosperma ramiflorum. Phytomedicine, 19, 413-417.

Di Mavungu, J. D., Malysheva, S. V., Sanders, M., Larionova, D., Robbens, J., Dubruel, P., et al. (2012). Development and validation of a new LC-MS/MS method for the simultaneous determination of six major ergot alkaloids and their corresponding epimers. Application to some food and feed commodities. Food Chemistry, 135, 292-303.

Dubrovay, Z., Háda, V., Béni, Z., \& Szántay, C., Jr. (2012). NMR and mass spectrometric characterization of vinblastine, vincristine and some new related impurities-Part I. Journal of Pharmaceutical and Biomedical Analysis, http://dx.doi.org/10.1016/j.jpba.2012.08.019. 
Duverneuil, C., de la Grandmaison, G. L., de Mazancourt, P., \& Alvarez, J. C. (2004). Liquid chromatography/photodiode array detection for determination of strychnine in blood: A fatal case report. Forensic Science International, 141, 17-21.

Etse, J. T., Gray, A. I., Thomas, D. W., \& Waterman, P. G. (1989). Terpenoid and alkaloid compounds from the seeds of Monodora brevipes. Phytochemistry, 28, 2489-2492.

Fang, L., Liu, Y., Yang, B., Wang, X., \& Huang, L. (2011). Separation of alkaloids from herbs using high-speed counter-current chromatography. Journal of Separation Science, 34, $2545-2558$.

Favretto, D., Piovan, A., Filippini, R., \& Caniato, R. (2001). Monitoring the production yields of vincristine and vinblastine in Catharanthus roseus from somatic embryogenesis. Semiquantitative determination by flow-injection electrospray ionization mass spectrometry. Rapid Communications in Mass Spectrometry, 15, 364-369.

Feng, H. T., Yuan, L. L., \& Li, S. F. Y. (2003). Analysis of Chinese medicine preparations by capillary electrophoresis-mass spectrometry. Journal of Chromatography. A, 1014, 83-91.

Ferreres, F., Pereira, D. M., Valentao, P., Oliveira, J. M. A., Faria, J., Gaspar, L., et al. (2010). Simple and reproducible HPLC-DAD-ESI-MS/MS analysis of alkaloids in Catharanthus roseus roots. Journal of Pharmacentical and Biomedical Analysis, 51, 65-69.

Fiot, J., Baghdikian, B., Boyer, L., Mahiou, V., Azas, N., Gasquet, M., et al. (2005). HPLC quantification of uncarine $\mathrm{D}$ and the anti-plasmodial activity of alkaloids from leaves of Mitragyna inermis (Willd.) O. Kuntze. Phytochemical Analysis, 16, 30-33.

Forseth, R. R., \& Schroeder, F. C. (2011). NMR-spectroscopic analysis of mixtures: From structure to function. Current Opinion in Chemical Biology, 15, 38-47.

Frach, K., \& Blaschke, G. (1998). Separation of ergot alkaloids and their epimers and determination in sclerotia by capillary electrophoresis. Journal of Chromatography. A, 808, $247-252$.

Frederich, M., Choi, Y. H., Angenot, L., Harnischfeger, G., Lefeber, A. W. M., \& Verpoorte, R. (2004). Metabolomic analysis of Strychnos nux-vomica, Strychnos icaja and Strychnos ignatii extracts by ${ }^{1} \mathrm{H}$ nuclear magnetic resonance spectrometry and multivariate analysis techniques. Phytochemistry, 65, 1993-2001.

Frederich, M., Choi, Y. H., \& Verpoorte, R. (2003). Quantitative analysis of strychnine and brucine in Strychnos nux-vomica using ${ }^{1} \mathrm{H}-\mathrm{NMR}$. Planta Medica, 69, 1169-1171.

Gallagher, C. A., Hough, L. B., Keefner, S. M., Seyed-Mozaffari, A., Archer, S., \& Glick, S. D. (1995). Identification and quantification of the indole alkaloid ibogaine in biological samples by gas chromatography-mass spectrometry. Biochemical Pharmacolog $\gamma, 49,73-79$.

Ganzera, M. (2008). Quality control oh herbal medicines by capillary electrophoresis: Potential, requirements and applications. Electrophoresis, 29, 3489-3503.

Ghosal, S., \& Srivastava, R. S. (1974). Structure of erysophorine: A new quaternary alkaloid of Erythrina arborescens. Phytochemistry, 13, 2603-2605.

Girardot, M., Deregnaucourt, C., Deville, A., Dubost, L., Joyeau, R., Allorge, L., et al. (2012). Indole alkaloids from Muntafara sessilifolia with antiplasmodial and cytotoxic activities. Phytochemistry, 73, 65-73.

Gonçalves, M. S., Curcino, I. J., Oliveira, R. R., \& Braz-Filho, R. (2011). Application of preparative high-speed counter-current chromatography for the separation of two alkaloids from the roots of Tabernaemontana catharinensis (Apocynaceae). Molecules, 16, $7480-7487$.

Gotti, R. (2011). Capillary electrophoresis of phytochemical substances in herbal drugs and medicinal plants. Journal of Pharmacentical and Biomedical Analysis, 55, 775-801.

Gu, X., Li, H., Zhu, R., \& Zou, H. (2006). Determination of strychnine and brucine in Strychnos nux-vomica L. by nonaqueous capillary electrophoresis. Chromatographia, 63, 289-292. 
Guilhaumou, R., Solas, C., Rome, A., Giocanti, M., Andre, N., \& Lacarelle, B. (2010). Validation of an electrospray ionization LC/MS/MS method for quantitative analysis of vincristine in human plasma samples. Journal of Chromatography B, 878, 423-427.

Guimaraes, H. A., Braz-Filho, R., \& Vieira, I. J. (2012). ${ }^{1} \mathrm{H}$ and ${ }^{13} \mathrm{C}-\mathrm{NMR}$ data of the simplest plumeran indole alkaloids isolated from Aspidosperma species. Molecules, 17, 3025-3043.

Háda, V., Dubrovay, Z., Lakó-Futó, A., Galambos, J., Gulyás, Z., \& Aranyi, A. (2012). NMR and mass spectrometric characterization of vinblastine, vincristine and some new related impurities-Part II. Journal of Pharmacentical and Biomedical Analysis, http://dx.doi.org/10.1016/j.jpba.2012.09.008.

He, L., Yang, L., Tan, R., Zhao, S., \& Hu, Z. (2011). Enhancement of vindoline production in suspension culture of the Catharanthus roseus cell line C20hi by light and methyl jasmonate elicitation. Analytical Sciences, 27, 1243-1248.

He, L., Yang, L., Xiong, A., Zhao, S., Wang, Z., \& Hu, Z. (2011). Simultaneous quantification of four indole alkaloids in Catharanthus roseus cell line C20hi by UPLC-MS. Analytical Sciences, 27, 433-438.

Hearn, W. L., Pablo, J., Hime, G. W., \& Mash, D. C. (1995). Identification and quantitation of ibogaine and an o-demethylated metabolite in brain and biological fluids using gas chromatography-mass spectrometry. Journal of Analytical Toxicology, 19, 427-434.

Henion, J. D., Mordehai, A. V., \& Cai, J. (1994). Quantitative capillary electrophoresis - Ion spray mass spectrometry on a benchtop ion trap for the determination of isoquinoline alkaloids. Analytical Chemistry, 66, 2103-2109.

Hermans-Lokkerbol, A., \& Verpoorte, R. (1986). Droplet counter-current chromatography of alkaloids. The influence of $\mathrm{pH}$-gradients and ion-pair formation on the retention of alkaloids. Planta Medica, 4, 299-302.

Herrero, M., Mendiola, J. A., Cifuentes, A., \& Ibañez, E. (2010). Supercritical fluid extraction: Recent advances and applications. Journal of Chromatography. A, 1217, 2495-2511.

Hesse, M. (1974). Indolalkaoide. In H. Budzikiewicz (Ed.), Progress in mass spectrometry. Teil 1: Text, Vol. 1. Weinheim, Germany: Verlag Chemie GmbH.

Heyman, H. M., \& Meyer, J. J. M. (2012). NMR-based metabolomics as a quality control tool for herbal products. South African Journal of Botany, 82, 21-32.

Holmes, E., Tang, H., Wang, Y., \& Seger, C. (2006). The assessment of plant metabolite profiles by NMR-based methodologies. Planta Medica, 72, 771-785.

Hong, B., Cheng, W., Wu, C., \& Zhao, C. (2010). Screening and identification of many of the compounds present in Rauvolfia verticillata by use of high-pressure LC and quadrupole TOF MS. Chromatographia, 72, 841-847.

Hou, Y., Cao, X., Wang, L., Cheng, B., Dong, L., Luo, X., et al. (2012). Microfractionation bioactivity-based ultra performance liquid chromatography/quadrupole time-of-flight mass spectrometry for the identification of nuclear factor- $\mathrm{kB}$ inhibitors and $\beta 2$ adrenergic receptors agonists in an alkaloidal extract of the folk herb Alstonia scholaris. Journal of Chromatography B, 908, 98-104.

Hu, W. L., Zhu, J. P., Prewo, R., \& Hesse, M. (1989). Alstogustine and 19-epialstogustine, quaternary indole alkaloids from Alstonia angustifolia. Phytochemistry, 28, 1963-1966.

Ingkaninan, K., Hazekamp, A., Hoek, A. C., Balconi, S., \& Verpoorte, R. (2000). Application of centrifugal partition chromatography in a general separation and dereplication procedure for plant extracts. Journal of Liquid Chromatography \& Related Technologies, 23, 2195-2208.

Ingkaninan, K., Hermans-Lokkerbol, A., \& Verpoorte, R. (1999). Comparison of some centrifugal partition chromatography systems for a general separation of plant extracts. Journal of Liquid Chromatography \& Related Technologies, 22, 885-896.

Ito, Y., \& Ma, Y. (1996). pH-zone-refining counter-current chromatography. Journal of Chromatography. A, 753,1-36. 
Jenett-Siems, K., Weigl, R., Kaloga, M., Schulz, J., \& Eich, E. (2003). Ipobscurines C and D: Macrolactam-type indole alkaloids from the seeds of Ipomoea obscura. Phytochemistry, 62, 1257-1263.

Jenks, C. (2002). Extraction studies of Tabernanthe iboga and Voacanga africana. Natural Product Letters, 16, 71-76.

Kaiser, C. S., Rompp, H., \& Schmidt, P. C. (2001). Pharmaceutical applications of supercritical carbon dioxide. Pharmazie, 56, 907-926.

Kingston, D. G. I. (1979). High performance liquid chromatography of natural products. Journal of Natural Products, 42, 237-260.

Kontrimaviciute, V., Breton, H., Mathieu, O., Mathieu-Daudé, J. C., \& Bressolle, F. M. M. (2006). Liquid chromatography-electrospray mass spectrometry determination of ibogaine and noribogaine in human plasma and whole blood. Application to a poisoning involving Tabernanthe iboga root. Journal of Chromatography B, 843, 131-141.

Kumar, V., Bulumulla, H. N. K., Wimalasiri, W. R., \& Reisch, J. (1994). Coumarins and an indole alkaloid from Pamburus missionis. Phytochemistry, 36, 879-881.

Lee, M. C., Chuang, W. C., \& Sheu, S. J. (1996). Determination of the alkaloids in Evodiae fructus by capillary electrophoresis. Journal of Chromatography. A, 755, 113-119.

Lee, H., Hong, W. H., Yoon, J. H., Song, K. M., Kwak, S. S., \& Liu, J. R. (1992). Extraction of indole alkaloids from Catharanthus roseus by using supercritical carbon dioxide. Biotechnology Techniques, 6, 127-130.

Li, Y., He, X., Qi, A., Gao, W., Chen, X., \& Hu, Z. (2006). Separation and determination of strychnine and brucine in Strychnos nux-vomica L. and its preparation by nonaqueous capillary electrophoresis. Journal of Pharmaceutical and Biomedical Analysis, 41, 400-407.

Li, C. Y., Lin, C. H., \& Wu, T. S. (2005). Quantitative analysis of camptothecin derivatives in Nothapodytes foetida using ${ }^{1} \mathrm{H}-\mathrm{NMR}$ method. Chemical and Pharmaceutical Bulletin, 53, 347-349.

Li, Y., Zhang, H., Hu, J. T., Xue, F., Li, Y. X., \& Sun, C. J. (2012). A GC-EI-MS-MS method for simultaneous determination of seven adulterants in slimming functional foods. Journal of Chromatographic Science, 50, 928-933.

Lindon, J. C., \& Nicholson, J. K. (2008). Analytical technologies for metabonomics and metabolomics, and multi-omic information recovery. Trends in Analytical Chemistry, 27, 194-204.

Liu, B., Guo, F., Chang, Y., Jiang, H., \& Wang, Q. (2010). Optimization of extraction of evodiamine and rutaecarpine from fruit of Evodia rutaecarpa using modified supercritical $\mathrm{CO}_{2}$. Journal of Chromatography. A, 1217, 7833-7839.

Liu, Y., Zhu, R., Li, H., Yan, M., \& Lei, Y. (2011). Ultra-performance liquid chromatography-tandem mass spectrometric method for the determination of strychnine and brucine in mice plasma. Journal of Chromatography B, 879, 2714-2719.

Lopez, C., Claude, B., Morin, Ph., Max, J. P., Pena, R., \& Ribet, J. P. (2011). Synthesis and study of a molecularly imprinted polymer for the specific extraction of indole alkaloids from Catharanthus roseus extracts. Analytica Chimica Acta, 683, 198-205.

Lopez-Avila, V., Benedicto, J., \& Robaugh, D. (1997). Supercritical fluid extraction of oxindole alkaloids from Uncaria tormentosa. Journal of High Resolution Chromatography, 20, 231-236.

Lorenz, N., Olsovska, J., Sulc, M., \& Tudzynski, P. (2010). Alkaloid cluster gene $\operatorname{ccs} A$ of ergot fungus Claviceps purpurea encodes chanoclavine I synthase, a flavin adenine dinucleotide-containing oxidoreductase mediating the transformation of N-methyldimethylallyltryptophan to chanocalvine I. Applied and Environmental Microbiology, 76, $1822-1830$.

Ma, C. H., Wang, S. Y., Yang, L., Zu, Y. G., Yang, F. J., Zhao, C. J., et al. (2012). Ionic liquid-aqueous solution ultrasonic-assisted extraction of camptothecin and 
10-hydroxtcamptothecin from Camptotheca acuminata samara. Chemical Engineering and Processing: Process Intensification, 57-58, 59-64.

Maltese, F., van der Kooy, F., \& Verpoorte, R. (2009). Solvent derived artifacts in natural products chemistry. Natural Products Communications, 4, 447-454.

Marques, E. P., Gil, F., Proença, P., Monsanto, P., Oliveira, M. F., Castanheira, A., et al. (2000). Analytical method for the determination of strychnine in tissues by gas chromatography/mass spectrometry: Two case reports. Forensic Science International, 110, 145-152.

Mbeunkui, F., Grace, M. H., \& Lila, M. A. (2012). Isolation and structural elucidation of indole alkaloids from Geissospermum vellosii by mass spectrometry. Journal of Chromatography $B, 885-886,83-89$.

McCalley, D. V. (2002). Analysis of the Cinchona alkaloids by high-performance liquid chromatography and other separation techniques. Journal of Chromatography. A, 967, $1-19$.

Mendiola, J. A., Herrero, M., Cifuentes, A., \& Ibañez, A. (2007). Use of compressed fluids for sample preparation: Food applications. Journal of Chromatography. A, 1152, 234-246.

Miao, P., Cai, D. C., Xiang, B. R., An, D. K., \& Ito, Y. (1998). Separation and purification of strychnine from crude extract of Strychnos nux-vomica L. By high-speed countercurrent chromatography. Journal of Liquid Chromatography \& Related Technologies, 21, 163-170.

Montoro, P., Carbone, V., Zuniga-Quiroz, J., De Simone, F., \& Pizza, C. (2004). Identification and quantification of components in extracts of Uncaria tomentosa by HPLC-ES/ MS. Phytochemical Analysis, 15, 55-64.

Moreno, P. R. H., van der Heijden, R., \& Verpoorte, R. (1993). Effect of terpenoid precursor feeding and elicitation on formation of indole alkaloids in cell suspension cultures of Catharanthus roseus. Plant Cell Reports, 12, 702-705.

Nguyen, D. T. T., Guillarme, D., Rudaz, S., \& Veuthey, J. L. (2006). Fast analysis in liquid chromatography using small particle size and high pressure. Journal of Separation Science, 29, 1836-1848.

Nielsen, H. B., Hazell, A., Hazell, R., Ghia, F., \& Torssell, K. B. G. (1994). Indole alkaloids and terpenoid from Tabernaemontana markgrafiana. Phytochemistry, 37, 1729-1735.

Niessen, W. M. A., Tjaden, U. R., \& van der Greef, J. (1993). Capillary electrophoresis-mass spectrometry. Journal of Chromatography, 636, 3-19.

Pallant, C. A., Cromarty, A. D., \& Steenkamp, V. (2012). Effect of an alkaloidal fraction of Tabernaemontana elegans (Stapf.) on selected micro-organisms. Journal of Ethnopharmacology, 140, 398-404.

Paranhos, J. T., Fragoso, V., da Silveira, V. C., Henriques, A. T., \& Fett-Neto, A. G. (2009). Organ-specific and environmental accumulation of psychollatine, a major indole alkaloid glucoside from Psychotria umbellate. Biochemical Systematics and Ecology, 37, 707-715.

Pedersen-Bjergaard, S., Rasmussen, K. E., \& Sannes, E. (1998). Strategies for the capillary electrophoretic separation of indole alkaloids in Psilocybe semilanceata. Electrophoresis, 19, 27-30.

Penelle, J., Christen, P., Molgó, J., Tits, M., Brandt, V., Frederich, M., et al. (2001). 5' ,6'Dehydroguiachrysine and $5^{\prime}, 6^{\prime}$-dehydroguiaflavine, two curarizing quaternary indole alkaloids from the stem bark of Strychnos guianensis. Phytochemistry, 58, 619-626.

Penelle, J., Tits, M., Christen, P., Molgo, J., Brandt, V., Frederich, M., et al. (2000). Quaternary indole alkaloids from the stem bark of Strychnos guianensis. Phytochemistry, 53, 1057-1066.

Pereira, C. G., Marques, M. O. M., Barreto, A. S., Siani, A. C., Fernandes, E. C., \& Meireles, M. A. M. (2004). Extraction of indole alkaloids from Tabernaemontana catharinensis using supercritical $\mathrm{CO} 2+$ ethanol: An evaluation of the process variables and the raw material origin. Journal of Supercritical Fluids, 30, 51-61.

Perera, P., Samuelson, G., van Beek, T. A., \& Verpoorte, R. (1983). Tertiary indole alkaloids from leaves of Tabernaemontana dichotoma. Planta Medica, 47, 148-150. 
Philipp, A. A., Wissenbach, D. K., Zoerntlein, S. W., Klein, O. N., Kanogsunthornrat, J., \& Maurer, H. H. (2009). Studies on the metabolism of mitragynine, the main alkaloid of the herbal drug Kratom, in rat and human urine using liquid chromatography-linear ion trap mass spectrometry. Journal of Mass Spectrometry, 44, 1249-1261.

Posch, T. N., Martin, N., Pütz, M., \& Huhn, C. (2012). Nonaqueous capillary electrophoresis-mass spectrometry: A versatile, straightforward tool for the analysis of alkaloids from psychoactive plant extracts. Electrophoresis, 33, 1557-1566.

Posch, T. N., Müller, A., Schulz, W., Pütz, M., \& Huhn, C. (2012). Implementation of a design of experiments to study the influence of the background electrolyte on separation and detection in non-aqueous capillary electrophoresis-mass spectrometry. Electrophoresis, 33, 583-598.

Quetin-Leclercq, J., Llabres, G., Warin, R., Belem-Pinheiro, M. L., Mavar-Manga, H., \& Angenot, L. (1995). Guianensine, a zwitterionic alkaloid from Strychnos guianensis. Phytochemistry, 40, 1557-1560.

Ramautar, R., Somsen, G. W., \& de Jong, G. J. (2011). CE-MS for metabolomics: Developments and applications in the period 2010-2012. Electrophoresis, 34, 86-98.

Ramírez, J., Ogan, K., \& Ratain, M. J. (1997). Determination of Vinca alkaloids in human plasma by liquid chromatography/atmospheric pressure chemical ionization mass spectrometry. Cancer Chemotherapy and Pharmacology, 39, 286-290.

Rasoanaivo, P., Martin, M. T., Guittet, E., \& Frappier, F. (2002). New contributions to the structure elucidation and pharmacology of Strychnos alkaloids. In Atta-urRahman (Ed.), Bioactive natural products: Studies in natural products chemistry: Vol. 26, Part G, (pp. 1029-1072). Elsevier BV.

Renault, J. H., Nuzillard, J. M., Le Crouerour, G., Thepenier, P., Zeches-Hanrot, M., \& Le Men-Olivier, L. (1999). Isolation of indole alkaloids from Catharanthus roseus by centrifugal partition chromatography in the $\mathrm{pH}$-zone refining mode. Journal of Chromatography. A, 849, 421-431.

Rosano, T. G., Hubbard, J. D., Meola, J. M., \& Swift, T. A. (2000). Fatal strychnine poisoning: Application of gas chromatography and tandem mass spectrometry. Journal of Analytical Toxicology, 24, 642-647.

Scriba, H. K. (2007). Non-aqueous capillary electrophoresis-mass spectrometry. Journal of Chromatography. A, 1159, 28-41.

Severino, V. G. P., Cazal, C. M., Forim, M. R., Silva, M. G. F., Rodrigues-Filho, E. R., Fernandes, J. B., et al. (2009). Isolation of secondary metabolites from Hortia oreadica (Rutaceae) leaves through high-speed counter-current chromatography. Journal of Chromatography. A, 1216, 4275-4281.

Sheludko, Y., Gerasimenko, I., Unger, M., Kostenyuk, I., \& Stoeckgit, J. (1999). Induction of alkaloid diversity in hybrid plant cell cultures. Plant Cell Reports, 18, 911-918.

Silvestrini, A., Pasqua, G., Botta, B., Monacelli, B., van der Heijden, R., \& Verpoorte, R. (2002). Effects of alkaloid precursor feeding on a Camptotheca acuminate cell line. Plant Physiology and Biochemistry, 40, 749-753.

Sohajda, T., Varga, E., Ivanyi, R., Fejos, I., Szente, L., Noszal, B., et al. (2010). Separation of vinca alkaloid enantiomers by capillary electrophoresis applying cyclodextrin derivatives and characterization of cyclodextrin complexes by nuclear magnetic resonance spectroscopy. Journal of Pharmaceutical and Biomedical Analysis, 53, 1258-1266.

Song, K. M., Park, S. W., Hong, W. H., Lee, H., Kwak, S. S., \& Liu, J. R. (1992). Isolation of vindoline from Catharanthus roseus by supercritical fluid extraction. Biotechnology Progress, 8, 583-586.

Sparr, C., \& Bjorklund, E. (2000). Analytical-scale microwave-assisted extraction. Journal of Chromatography. A, 902, 227-250.

Srivastava, A., Tripathi, A. K., Pandey, R., Verma, R. K., \& Gupta, M. M. (2006). Quantitative determination of reserpine, ajmaline, and ajmalicine in Rauvolfia serpentina by 
reversed-phase high-performance liquid chromatography. Journal of Chromatographic Science, 44, 557-560.

Stöckigt, J., Sheludko, Y., Unger, M., Gerasimenko, I., Warzecha, H., \& Stöckigt, D. (2002). High-performance liquid chromatographic, capillary electrophoretic and capillary electrophoretic-electrospray ionisation mass spectrometric analysis of selected alkaloid groups. Journal of Chromatography. A, 967, 85-113.

Stöckigt, D., Unger, M., Belder, D., \& Stöckigt, J. (1997). Analysis of Rauwolfia alkaloids employing capillary electrophoresis-mass spectrometry. Natural Product Letters, 9, 265-272.

Stuppner, H., Sturn, S., \& Konwalinka, G. (1992). Capillary electrophoretic analysis of oxindole alkaloids from Uncaria tomentosa. Journal of Chromatography, 609, 375-380.

Sun, J., \& Chen, P. (2012). Chromatographic fingerprint analysis of yohimbe bark and related dietary supplements using UHPLC/UV/MS. Journal of Pharmaceutical and Biomedical Analysis, 61, 142-149.

Sun, C., \& Liu, H. (2008). Application of non-ionic surfactant in the microwave-assisted extraction of alkaloids from Rhizoma Coptidis. Analytica Chimica Acta, 612, 160-164.

Talapatra, B., Patra, A., \& Talapatra, S. K. (1975). Terpenoids and alkaloids of the leaves of Tabernaemontana coronaria. Phytochemistry, 14, 1652-1653.

Tanaka, J. C. A., da Silva, C. C., Ferreira, I. C. P., Machado, G. M. C., Leon, L. L., \& de Oliveira, A. J. B. (2007). Antileshmanial activity of indole alkaloids from Aspidosperma. Phytomedicine, 14, 377-380.

Unger, M. (2009). Capillary electrophoresis of natural products: Current applications and recent advances. Planta Medica, 75, 735-745.

Unger, M., Stöckigt, D., Belder, D., \& Stöckigt, J. (1997). General approach for the analysis of various alkaloid classes using capillary electrophoresis and capillary electrophoresismass spectrometry. Journal of Chromatography. A, 767, 263-276.

van der Heijden, R., Hermans-Lokkerbol, A., Verpoorte, R., \& Baerheim Svendsen, A. (1987). Pharmacognostical studies on Tabernaemontana XX. Ion-pair droplet countercurrent chromatography of indole alkaloids from suspension cultures. Journal of Chromatography, 396, 410-415.

Van Eenoo, P., Deventer, K., Roels, K., \& Delbeke, F. T. (2006). Quantitative LC-MS determination of strychnine in urine after ingestion of a Strychnos nux-vomica preparation and its consequences in doping control. Forensic Science International, 164, 159-163.

Verardo, V., Gomez-Caravaca, A. M., Seura-Carretero, A., Caboni, M. F., \& FernándezGutiérrez, A. (2011). Development of a CE-ESI-micro TOF-MS method for a rapid identification of phenolic compounds in buckwheat. Electrophoresis, 32, 669-673.

Verma, A., Hartonen, K., \& Riekkola, M. L. (2008). Optimization of supercritical fluid extraction of indole alkaloids from Catharanthus roseus using experimental design methodology-Comparison with other extraction techniques. Phytochemical Analysis, $19,52-63$.

Verma, A., Laakso, I., Seppanen-Lakso, T., Huhtikangas, A., \& Riekkola, M. L. (2007). A simplified procedure for indole alkaloid extraction from Catharanthus roseus combined with a semi-synthetic production process for vinblastine. Molecules, 12, 1307-1315.

Verpoorte, R. (2005). Alkaloids. In P. Worsfold, A. Townshend, \& C. Poole (Eds.), Encyclopedia of analytical science (pp. 56-61), 2nd ed. Elsevier.

Verpoorte, R., \& Baerheim Svendsen, A. (Eds.), (1984). Chromatography of alkaloids, part II, GLC and HPLC. In Chromatography library, Vol. 23B, (pp. 1-467). Amsterdam: Elsevier.

Verpoorte, R., Choi, Y. H., Mustafa, R. N., \& Kim, H. K. (2008). Metabolomics: Back to basics. Phytochemistry Reviews, 7, 525-538.

Verpoorte, R., \& Niessen, W. M. A. (1994). Liquid chromatography coupled with mass spectrometry in the analysis of alkaloids. Phytochemical Analysis, 5, 217-232. 
Vieira, I. J. C., Medeiros, W. L. B., Monnerat, C. S., Souza, J. J., Mathias, L., Braz-Filho, R., et al. (2008). Two fast screening methods (GC-MS and TLC-ChEI assay) for rapid evaluation of potential anticholinesterase indole alkaloids in complex mixtures. Annals of the Brazilian Academy of Sciences, 80, 419-426.

Wang, C., Han, D., Wang, Z., Zang, X., \& Wu, Q. (2006). Analysis of Strychnos alkaloids in traditional Chinese medicines with improved sensitivity by sweeping micellar electrokinetic chromatography. Analytica Chimica Acta, 572, 190-196.

Wang, P., Sun, H., Lv, H., Sun, W., Yuan, Y., Han, Y., et al. (2010). Thyroxine and reserpine-induced changes in metabolic profiles of rat urine and therapeutic effect of Liu Wei Di Huang Wan detected by UPLC-HDMS. Journal of Pharmacology and Biomedical Analysis, 53, 631-645.

Wang, C. H., Wang, G. C., Wang, Y., Zhang, X. Q., Huang, X. J., Zhang, D. M., et al. (2012). Cytotoxic dimeric indole alkaloids from Catharanthus roseus. Fitoterapia, 83, 765-769.

Wang, S. Y., Yang, L., Zu, Y. G., Zhao, C. J., Sun, X. W., Zhang, L., et al. (2011). Design and performance evaluation of ionic-liquids-based microwave-assisted environmentally friendly extraction technique for camptothecin and 10-hydroxycamptothecin from Samara of Camptotheca acuminata. Industrial and Engineering Chemistry Research, 50, 13620-13627.

Wang, J., Zheng, H., Efferth, T., Wang, R., Shen, Y., \& Hao, X. (2005). Indole and carbazole alkaloids from Glycosmis Montana with weak anti-HIV and cytotoxic activities. Phytochemistry, 66, 697-701.

Wen, D., Li, C., Liu, Y., Liao, Y., \& Liu, H. (2006). Determination of evodiamine and rutecarpine in human serum by liquid chromatography-tandem mass spectrometry. Analytical and Bioanalytical Chemistry, 385, 1075-1081.

Xie, J., Zhu, L., Luo, H., Zhou, L., Li, C., \& Xu, X. (2001). Direct extraction of specific pharmacophoric flavonoids from gingko leaves using a molecularly imprinted polymer for quercetin. Journal of Chromatography. A, 934, 1-11.

Xu, Y. J., Foubert, K., Dhooghe, L., Lemiere, F., Cimanga, K., Mesia, K., et al. (2012). Chromatographic profiling and identification of two new iridoid-indole alkaloids by UPLC-MS and HPLC-SPE-NMR analysis of an antimalarial extract from Nauclea pobenguinii. Phytochemistry Letters, 5, 316-319.

Yang, L., Wang, H., Zu, Y. G., Zhao, C., Zhang, L., Chen, X., et al. (2011). Ultrasoundassisted extraction of the three terpenoid indole alkaloids vindoline, catharanthine and vinblastine from Catharanthus roseus using ionic liquid aqueous solutions. Chemical Engineering Journal, 172, 705-712.

Yilmaz, A., Nyberg, N. T., \& Jaroszewski, J. W. (2012). Extraction of alkaloids for NMRbased profiling: Exploratory analysis of an archaic Cinchona bark collection. Planta Medica, 78, 1885-1890.

Zanolari, B., Ndjoko, K., Ioset, J. R., Marston, A., \& Hostettmann, K. (2003). Qualitative and quantitative determination $\mathrm{f}$ yohimbine in authentic yohimbe bark and in commercial aphrodisiacs by HPLC-UV-API/MS methods. Phytochemical Analysis, 14, 193-201.

Zhang, J., Yu, Y., Liu, D., \& Liu, Z. (2007). Extraction and composition of three naturally occurring anti-cancer alkaloids in Camptotheca acuminata seed and leaf extracts. Phytomedicine, 14, 50-56.

Zhao, C., \& He, C. (2006). Preparative isolation and purification of atractylon and atractylenolide III from the Chinese medicinal plant Atractylodes macrocephala by highspeed counter-current chromatography. Journal of Separation Science, 29, 1630-1636.

Zhou, H., Tai, Y., Sun, C., \& Pan, Y. (2005). Rapid identification of vinca alkaloids by direct-injection electrospray ionization tandem mass spectrometry and confirmation 
by high-performance liquid chromatography-mass spectrometry. Phytochemical Analysis, $16,328-333$.

Zhu, Q. H., Huang, D. D., Li, L., \& Yin, Y. G. (2010). Synthesis of molecularly imprinted polymers for the application of selective clean-up vinblastine from Catharanthus roseus extract. Science China Chemistry, 53, 2587-2592.

Zong, Y. Y., \& Che, C. T. (1995). Determination of strychnine and brucine by capillary zone electrophoresis. Planta Medica, 5, 456-458. 